Supporting Information

\title{
Ligand Coordination Site-Directed Assembly of Copper(I) Iodide Complexes of ((Pyridyl)-1-pyrazolyl)pyridine
}

Jun-Chi Li, ${ }^{\dagger}$ Hong-Xi Li, ${ }^{,}{ }^{\dagger}$ Hai-Yan Li, ${ }^{\dagger}$ Wei-Jie Gong, ${ }^{\dagger}$ and Jian-Ping Lang*, ${ }^{\dagger, \ddagger}$

${ }^{\dagger}$ State and Local Joint Engineering Laboratory for Novel Functional Polymeric Materials, College of Chemistry, Chemical Engineering and Materials Science, Soochow University, Suzhou 215123, Jiangsu, People’s Republic of China.

$¥$ State Key Laboratory of Organometallic Chemistry, Shanghai Institute of Organic Chemistry, Chinese Academy of Sciences, Shanghai 200032, People's Republic of China 


\section{Table of Contents}

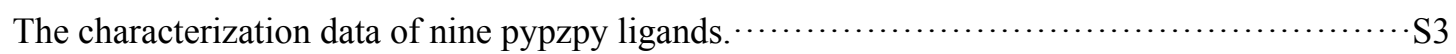

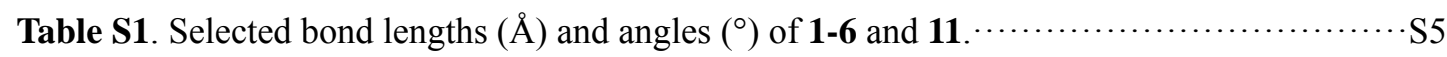

Figure S1. The UV-vis spectra of pypzpy ligands in $\mathrm{MeCN}$ in a 1-cm-thick glass cell. $\cdots \cdots \cdots \cdot \cdot \mathrm{S} 7$

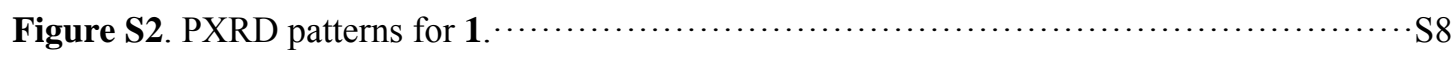

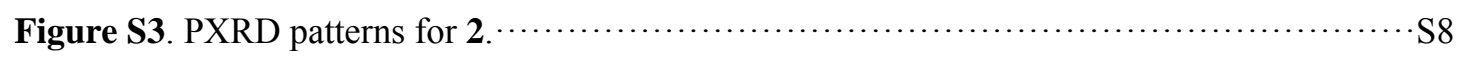

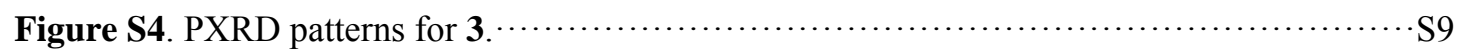

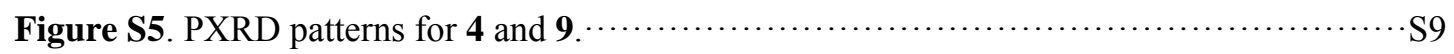

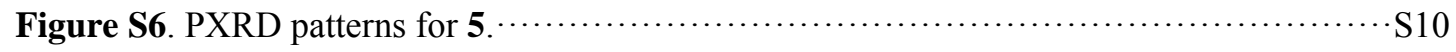

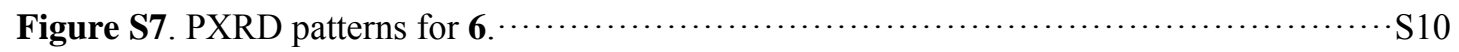

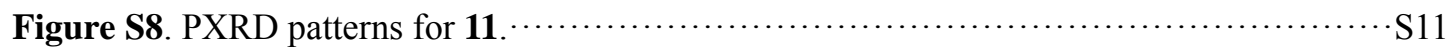

Figure S9. PXRD patterns for 8 and 10. …

Figure S10. View of the 2D hydrogen-bound structure of $1 . \cdots \cdots \cdots \cdots \cdots \cdots \cdots \cdots \cdots \cdots \cdots \cdots \cdots \cdots \cdots \cdots \cdots \cdots \cdots$

Figure S11. View of the molecular structure of 5 with a labeling scheme and $50 \%$ thermal

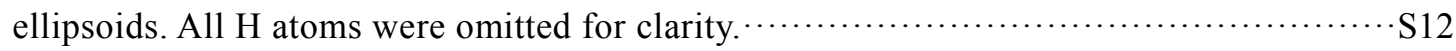

Figure S12. $\pi-\pi$ interactions between the two pyrazolyl rings and the two pyridyl rings of $2 . \cdot \mathrm{S} 13$

Figure S13. $\pi-\pi$ interactions between the two pyridyl rings of 3. $\cdots \cdots \cdots \cdots \cdots \cdots \cdots \cdots \cdots \cdots \cdots \cdots \cdots \cdots \cdots \cdots$

Figure S14. View of the 3D hydrogen-bound structure of $4 . \cdots \cdots \cdots \cdots \cdots \cdots \cdots \cdots \cdots \cdots \cdots \cdots \cdots \cdots \cdots \cdots \cdots \cdots \cdots \cdots$

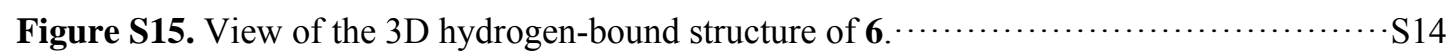

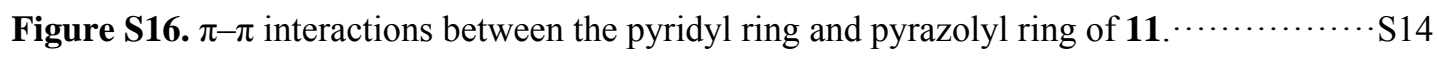

Figure S17. View of the 3D hydrogen-bound structure of $11, \cdots \cdots \cdots \cdots \cdots \cdots \cdots \cdots \cdots \cdots \cdots \cdots \cdots \cdots \cdots \cdots \cdots \cdots$

Figure S18. Emission spectra of pypzpy ligands in $\mathrm{MeCN}$ solution at room temperature. $\cdots \cdots \cdot \mathrm{S} 15$

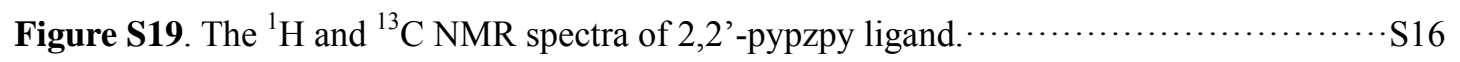

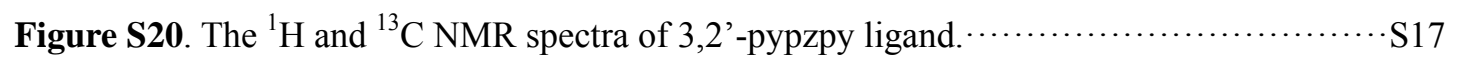

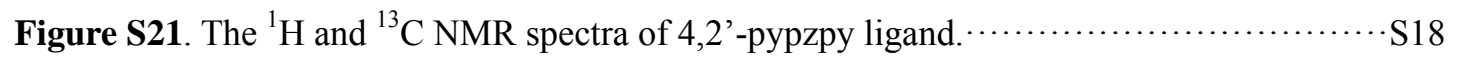

Figure S22. The ${ }^{1} \mathrm{H}$ and ${ }^{13} \mathrm{C}$ NMR spectra of 2,3'-pypzpy ligand. …...................... 19

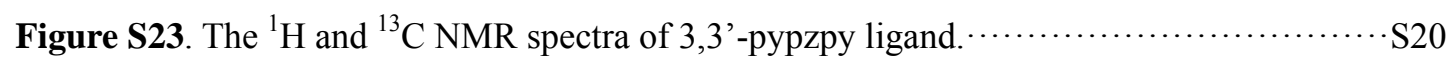

Figure S24. The ${ }^{1} \mathrm{H}$ and ${ }^{13} \mathrm{C}$ NMR spectra of 4,3'-pypzpy ligand. $\cdots \cdots \cdots \cdots \cdots \cdots \cdots \cdots \cdots \cdots \cdots \cdots \cdots \cdots \cdots \cdots \cdots \cdots \cdots$

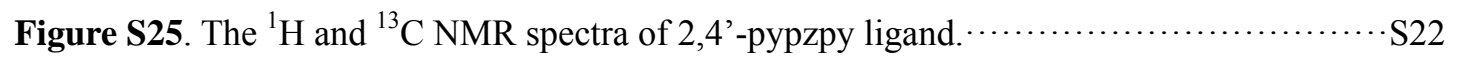

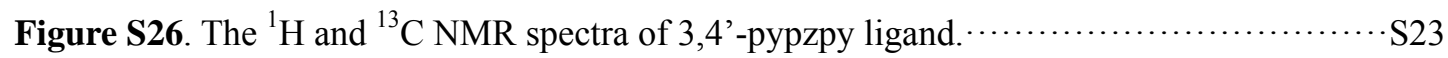

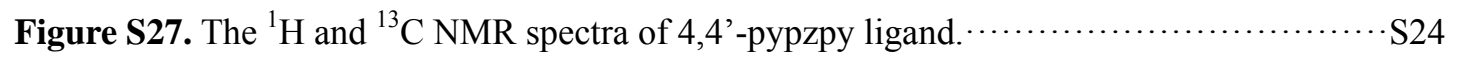




\section{The characterization data of nine pypzpy ligands}

2,2'-pypzpy: m.p. $258-260{ }^{\circ} \mathrm{C}$. Anal. Calcd for $\mathrm{C}_{13} \mathrm{H}_{10} \mathrm{~N}_{4}$ : C, 70.26; H, 4.54; N, 25.21\%. Found: $\mathrm{C}$, 70.22; H, 4.68; N, 24.58\%. ${ }^{1} \mathrm{H} \quad \mathrm{NMR}\left(400 \mathrm{MHz}, \mathrm{DMSO}-d_{6}, \mathrm{ppm}\right): \delta=8.73(\mathrm{~d}, J=4.0 \mathrm{~Hz}, 1 \mathrm{H})$, $8.66(\mathrm{~d}, J=4.0 \mathrm{~Hz}, 1 \mathrm{H}), 8.52(\mathrm{~d}, J=4.0 \mathrm{~Hz}, 1 \mathrm{H}), 8.14(\mathrm{~d}, J=8.0 \mathrm{~Hz}, 1 \mathrm{H}), 8.07$ (s, 2H), 7.93 (t, $J$ $=8.0 \mathrm{~Hz}, 1 \mathrm{H}), 7.41(\mathrm{t}, J=8.0 \mathrm{~Hz}, 2 \mathrm{H}), 7.14(\mathrm{~d}, J=4.0 \mathrm{~Hz}, 1 \mathrm{H}) ;{ }^{13} \mathrm{C}$ NMR $\left(100 \mathrm{MHz}\right.$, DMSO- $d_{6}$, ppm): $\delta=154.1,151.3,151.1,149.9,148.8,140.0,137.5,129.1,123.9,122.7,120.3,112.5,107.2$. HRMS (EI) $\mathrm{m} / z$ : Calcd for $\mathrm{C}_{13} \mathrm{H}_{10} \mathrm{~N}_{4}$ 222.0905; found 222.0907. IR (KBr disk): 1591 (s), 1577 (m), 1531 (m), 1482 (m), 1470 (s), 1450 (s), 1417 (m), 1366 (m), 1317 (m), 1279 (m), 1269 (m), 1146 (w), 1051 (m), 991 (m), 958 (m), 949 (m), 796 (m), 768 (s), 742 (m), 725 (m), $621(\mathrm{w}) \mathrm{cm}^{-1}$.

3,2'-pypzpy: Yield: $85 \%$ m.p. $232-234^{\circ} \mathrm{C}$. Anal. Calcd for $\mathrm{C}_{13} \mathrm{H}_{10} \mathrm{~N}_{4}: \mathrm{C}, 70.26 ; \mathrm{H}, 4.54 ; \mathrm{N}$, $25.21 \%$. Found: C, 69.53; H, 4.916; N, 24.34\%. ${ }^{1} \mathrm{H}$ NMR (400 MHz, DMSO- $d_{6}, \mathrm{ppm}$ ): $\delta=9.27$ (s, $1 \mathrm{H}), 8.75(\mathrm{~s}, 1 \mathrm{H}), 8.60(\mathrm{~s}, 1 \mathrm{H}), 8.51(\mathrm{~d}, J=4.0 \mathrm{~Hz}, 1 \mathrm{H}), 8.34(\mathrm{~d}, J=4.0 \mathrm{~Hz}, 1 \mathrm{H}), 8.06(\mathrm{~s}, 2 \mathrm{H})$, $7.52(\mathrm{t}, J=8.0 \mathrm{~Hz}, 1 \mathrm{H}), 7.41(\mathrm{~s}, 1 \mathrm{H}), 7.22(\mathrm{~s}, 1 \mathrm{H}) ;{ }^{13} \mathrm{C}$ NMR (100 MHz, DMSO- $\left.d_{6}, \mathrm{ppm}\right): \delta=$ $151.0,150.8,149.8,148.8,147.3,140.1,133.3,129.3,128.6,124.4,122.7,112.6,106.6$. HRMS (EI) $m / z$ : Calcd for $\mathrm{C}_{13} \mathrm{H}_{10} \mathrm{~N}_{4}$ 222.0905; found 222.0911. IR (KBr disk): 1595 (w), 1581 (m), 1530 (m), 1475 (s), 1456 (s), 1420 (m), 1368 (m), 1278 (w), 1063 (m), 992 (m), 959 (w), 774 (s), 752 (m), $694(\mathrm{~m}), 702(\mathrm{~m}), 668(\mathrm{~m}), 618(\mathrm{~m}), 512(\mathrm{w}) \mathrm{cm}^{-1}$.

4,2'-pypzpy: Yield: $82 \%$. m.p. $232-234^{\circ} \mathrm{C}$. Anal. Calcd for $\mathrm{C}_{13} \mathrm{H}_{10} \mathrm{~N}_{4}: \mathrm{C}, 70.26 ; \mathrm{H}, 4.54 ; \mathrm{N}$, $25.21 \%$. Found: C, $70.17 ; \mathrm{H}, 4.703 ; \mathrm{N}, 24.53 \%$. ${ }^{1} \mathrm{H}$ NMR (400 MHz, DMSO- $\left.d_{6}, \mathrm{ppm}\right): \delta=8.76$ (d, $J=4.0 \mathrm{~Hz}, 1 \mathrm{H}), 8.68(\mathrm{~d}, J=4.0 \mathrm{~Hz}, 2 \mathrm{H}), 8.52(\mathrm{~s}, J=4.0 \mathrm{~Hz}, 1 \mathrm{H}), 8.08(\mathrm{~s}, 2 \mathrm{H}), 7.94$ (d, $J=8.0$ $\mathrm{Hz}, 2 \mathrm{H}), 7.43(\mathrm{t}, J=4.0 \mathrm{~Hz}, 1 \mathrm{H}), 7.27(\mathrm{~d}, J=4.0 \mathrm{~Hz}, 1 \mathrm{H}) ;{ }^{13} \mathrm{C}$ NMR $\left(100 \mathrm{MHz}\right.$, DMSO- $\left.d_{6}, \mathrm{ppm}\right)$ : $\delta=151.1,151.0,150.8,148.9,140.2,139.9,129.6,122.9,120.4,112.7,107.2$. HRMS (EI) $\mathrm{m} / z$ : Calcd for $\mathrm{C}_{13} \mathrm{H}_{10} \mathrm{~N}_{4}$ 222.0905; found 222.0904. IR (KBr disk): 1597 (s), 1572 (m), 1527 (m), 1471 (s), 1458 (m), 1439 (m), 1418 (w), 1367 (s), 1306 (m), 1272 (m), 1219 (w), 1057 (m), 991 (m), $960(\mathrm{~m}), 831(\mathrm{~m}), 787(\mathrm{~m}), 766(\mathrm{~m}), 720(\mathrm{~s}), 686(\mathrm{w}), 621(\mathrm{w}), 521(\mathrm{w}) \mathrm{cm}^{-1}$.

3,2'-pypzpy: Yield: $85 \%$ m.p. $301-302^{\circ} \mathrm{C}$. Anal. Calcd for $\mathrm{C}_{13} \mathrm{H}_{10} \mathrm{~N}_{4}: \mathrm{C}, 70.26 ; \mathrm{H}, 4.54 ; \mathrm{N}$, $25.21 \%$. Found: $\mathrm{C}, 69.55 ; \mathrm{H}, 4.60 ; \mathrm{N}, 24.90 \% .{ }^{1} \mathrm{H}$ NMR (400 MHz, DMSO- $d_{6}, \mathrm{ppm}$ ): $\delta=9.24$ (s, $1 \mathrm{H}), 8.72-8.58(\mathrm{~m}, 3 \mathrm{H}), 8.36(\mathrm{~d}, J=8.0 \mathrm{~Hz}, 1 \mathrm{H}), 8.13(\mathrm{~d}, J=8.0 \mathrm{~Hz}, 1 \mathrm{H}), 7.92(\mathrm{t}, J=4.0 \mathrm{~Hz}, 1 \mathrm{H})$, $7.61(\mathrm{t}, J=4.0 \mathrm{~Hz}, 1 \mathrm{H}), 7.40(\mathrm{t}, J=4.0 \mathrm{~Hz}, 1 \mathrm{H}), 7.18(\mathrm{~s}, 1 \mathrm{H}) ;{ }^{13} \mathrm{C}$ NMR $\left(100 \mathrm{MHz}\right.$, DMSO- $d_{6}$, ppm): $\delta=153.3,150.8,149.4,147.3,139.9,137.0,135.9,130.0,125.8,124.2,123.3,119.8$, 106.9. HRMS (EI) $\mathrm{m} / z$ : Calcd for $\mathrm{C}_{13} \mathrm{H}_{10} \mathrm{~N}_{4}$ 222.0905; found 222.0912. IR (KBr disk): 1595 (s), 1583 (m), 1566 (w), 1495 (s), 1455 (s), 1415 (m), 1370 (s), 1388 (w), 1279 (s), 1234 (w), 1189 (w), $1140(\mathrm{w}), 1054$ (w), 993 (w), $942(\mathrm{~m}), 799(\mathrm{~m}), 756(\mathrm{~s}), 723$ (w), $703(\mathrm{~m}), 618(\mathrm{w}) \mathrm{cm}^{-1}$.

3,3'-pypzpy: Yield: 87 \%. m.p. $321-323^{\circ} \mathrm{C}$. Anal. Calcd for $\mathrm{C}_{13} \mathrm{H}_{10} \mathrm{~N}_{4}: \mathrm{C}, 70.26$; H, 4.54; N, $25.21 \%$. Found: C, 69.18; H, 4.63; N, 24.69\%. ${ }^{1} \mathrm{H}$ NMR (400 MHz, DMSO- $\left.d_{6}, \mathrm{ppm}\right): \delta=9.22(\mathrm{~d}$, $J=16.0 \mathrm{~Hz}, 2 \mathrm{H}), 8.75(\mathrm{~s}, 1 \mathrm{H}), 8.59(\mathrm{~s}, 2 \mathrm{H}), 8.34(\mathrm{t}, J=8.0 \mathrm{~Hz}, 2 \mathrm{H}), 7.62-7.52(\mathrm{~m}, 2 \mathrm{H}), 7.25(\mathrm{~s}$, $1 \mathrm{H}) ;{ }^{13} \mathrm{C}$ NMR (100 MHz, DMSO- $\left.d_{6}, \mathrm{ppm}\right): \delta=150.0,149.2,147.3,146.8,139.8,135.8,132.7$, 
130.1, 128.1, 125.7, 124.2, 123.9, 106.2. HRMS (EI) $\mathrm{m} / z$ : Calcd for $\mathrm{C}_{13} \mathrm{H}_{10} \mathrm{~N}_{4}$ 222.0905; found 222.0908. IR (KBr disk): 1630 (m), 1586 (s), 1526 (w), 1491 (s), 1452 (m), 1413 (w), 1383 (w), $1366(\mathrm{~m}), 1355$ (w), 1315 (w), $1275(\mathrm{~m}), 1236(\mathrm{w}), 1188(\mathrm{w}), 1173$ (w), $1138(\mathrm{~m}), 1056(\mathrm{~s}), 1024$ (w), $955(\mathrm{~m}), 807(\mathrm{w}), 781(\mathrm{~m}), 704(\mathrm{~m}), 625(\mathrm{~m}), 524(\mathrm{w}) \mathrm{cm}^{-1}$.

4,3'-pypzpy: Yield: $80 \%$ m.p. $280-282^{\circ} \mathrm{C}$. Anal. Calcd for $\mathrm{C}_{13} \mathrm{H}_{10} \mathrm{~N}_{4}: \mathrm{C}, 70.26 ; \mathrm{H}, 4.54 ; \mathrm{N}$, 25.21\%. Found: C, 70.02; H,4.67; N, 24.85\%. ${ }^{1} \mathrm{H}$ NMR (400 MHz, DMSO- $d_{6}, \mathrm{ppm}$ ): $\delta=9.24$ (s, $1 \mathrm{H}), 8.77$ (s, 1H), 8.69 (s, 2H), 8.60 (s, 1H), 8.36 (d, $J=8.0 \mathrm{~Hz}, 1 \mathrm{H}), 7.93(\mathrm{~s}, 2 \mathrm{H}), 7.63-7.60$ (m, $1 \mathrm{H}), 7.31(\mathrm{~s}, 1 \mathrm{H}) ;{ }^{13} \mathrm{C}$ NMR (100 MHz, DMSO- $\left.d_{6}, \mathrm{ppm}\right): \delta=150.3,150.2,147.6,140.0,139.3$, 135.7, 130.4, 125.9, 124.2, 119.8, 106.8. HRMS (EI) $\mathrm{m} / z$ : Calcd for $\mathrm{C}_{13} \mathrm{H}_{10} \mathrm{~N}_{4} 222.0905$; found 222.0901. IR (KBr disk): 1604 (s), 1583 (m), 1505 (s), 1448 (m), 1392 (w), 1370 (m), 1357 (w), 1309 (m), 1278 (w), 1252 (w), 1219 (w), $1125(\mathrm{~m}), 1055(\mathrm{~m}), 1021(\mathrm{w}), 990(\mathrm{w}), 961(\mathrm{~m}), 944$ (w), $831(\mathrm{~m}), 804(\mathrm{~m}), 763(\mathrm{~s}), 722(\mathrm{w}), 700(\mathrm{w}), 689(\mathrm{~m}), 618(\mathrm{~m}), 517(\mathrm{w}) \mathrm{cm}^{-1}$.

2,4'-pypzpy: Yield: $85 \%$. m.p. $345-347^{\circ}$ C. Anal. Calcd for $\mathrm{C}_{13} \mathrm{H}_{10} \mathrm{~N}_{4}$ : C, 70.26; H, 4.54; N, 25.21\%. Found: C, 70.07; H, 4.61; N, 24.84\%. ${ }^{1} \mathrm{H}$ NMR (400 MHz, DMSO- $d_{6}$, ppm): $\delta=8.88$ (s, $1 \mathrm{H}), 8.77-8.73(\mathrm{~m}, 3 \mathrm{H}), 8.20(\mathrm{~d}, J=8.0 \mathrm{~Hz}, 1 \mathrm{H}), 8.05-7.97(\mathrm{~m}, 3 \mathrm{H}), 7.47(\mathrm{t}, J=4.0 \mathrm{~Hz}, 1 \mathrm{H}), 7.27$ $(\mathrm{d}, J=4.0 \mathrm{~Hz}, 1 \mathrm{H}) ;{ }^{13} \mathrm{C}$ NMR $\left(100 \mathrm{MHz}\right.$, DMSO- $\left.d_{6}, \mathrm{ppm}\right): \delta=153.9,151.2,150.6,149.5,145.3$, 137.1, 130.1, 123.6, 120.0, 112.2, 107.6. HRMS (EI) $m / z$ : Calcd for $\mathrm{C}_{13} \mathrm{H}_{10} \mathrm{~N}_{4} 222.0905$; found 222.0905. IR (KBr disk): 1589 (s), 1533 (w), 1506 (w), 1484 (m), 1459 (w), 1420 (w), 1381 (m), 1342 (m), 1281 (m), 1049 (m), 991 (w), $824(\mathrm{~m}), 760$ (m), $716(\mathrm{w}), 688(\mathrm{~m}), 622(\mathrm{w}), 526(\mathrm{w})$ $\mathrm{cm}^{-1}$.

3,4'-pypzpy: Yield: 88 \%. m.p. $238-240^{\circ} \mathrm{C}$. Anal. Calcd for $\mathrm{C}_{13} \mathrm{H}_{10} \mathrm{~N}_{4}$ : C, 70.26; H, 4.54; N, $25.21 \%$. Found: C, 70.03; H, 4.68; N, 24.97\%. ${ }^{1} \mathrm{H}$ NMR (400 MHz, DMSO- $d_{6}, \mathrm{ppm}$ ): $\delta=9.22$ (s, $1 \mathrm{H}), 8.86(\mathrm{~d}, J=4.0 \mathrm{~Hz}, 1 \mathrm{H}), 8.73-8.63(\mathrm{~m}, 3 \mathrm{H}), 8.35(\mathrm{~d}, J=8.0 \mathrm{~Hz}, 1 \mathrm{H}), 7.99(\mathrm{~d}, J=4.0 \mathrm{~Hz}, 2 \mathrm{H})$, $7.53(\mathrm{t}, J=4.0 \mathrm{~Hz}, 1 \mathrm{H}), 7.30(\mathrm{~s}, 1 \mathrm{H}) ;{ }^{13} \mathrm{C}$ NMR (100 MHz, DMSO- $\left.d_{6}, \mathrm{ppm}\right): \delta=151.1,150.6$, 149.4, 146.9, 145.1, 132.9, 130.2, 127.8, 123.8, 112.1, 106.9. HRMS (EI) $m / z$ : Calcd for $\mathrm{C}_{13} \mathrm{H}_{10} \mathrm{~N}_{4}$ 222.0905; found 222.0909. IR (KBr disk): 1592 (s), 1530 (w), 1505 (w), 1426 (w), 1366 (w), 1322 (w), $1317(w), 1284(w), 1216(w), 1057(w), 992(w), 954(w), 818(w), 787(w), 721(w), 706$ (w), $691(\mathrm{w}) \mathrm{cm}^{-1}$.

4,4'-pypzpy: Yield: 90 \%. m.p. $363-364^{\circ} \mathrm{C}$. Anal. Calcd for $\mathrm{C}_{13} \mathrm{H}_{10} \mathrm{~N}_{4}: \mathrm{C}, 70.26 ; \mathrm{H}, 4.54 ; \mathrm{N}$, 25.21\%. Found: C, 70.12; H, 4.60; N, 25.24\%. ${ }^{1} \mathrm{H}$ NMR (400 MHz, DMSO- $\left.d_{6}, \mathrm{ppm}\right): \delta=8.88$ (d, $J=4.0 \mathrm{~Hz}, 1 \mathrm{H}), 8.70(\mathrm{~s}, 4 \mathrm{H}), 7.99-7.93(\mathrm{~m}, 4 \mathrm{H}), 7.35(\mathrm{~d}, J=4.0 \mathrm{~Hz}, 1 \mathrm{H}) ;{ }^{13} \mathrm{C}$ NMR $(100 \mathrm{MHz}$, DMSO- $\left.d_{6}, \mathrm{ppm}\right): \delta=151.2,150.9,150.2,145.1,130.6,130.6,120.0,112.3,107.5$. HRMS (EI) $m / z$ : Calcd for $\mathrm{C}_{13} \mathrm{H}_{10} \mathrm{~N}_{4}$ 222.0905; found 222.0913. IR (KBr disk): 1590 (s), 1528 (w), 1509 (m), 1423 (m), 1395 (w), $1384(w), 1375(w), 1343(w), 1308(w), 1282(w), 1211(w), 1055(w), 993$ (w), $961(\mathrm{w}), 819(\mathrm{~m}), 761(\mathrm{~m}), 708(\mathrm{~m}), 690(\mathrm{~m}), 682(\mathrm{w}), 515(\mathrm{w}) \mathrm{cm}^{-1}$. 
Table S1. Selected bond lengths $(\AA)$ and angles $\left(^{\circ}\right)$ of 1-6 and 11

\begin{tabular}{|c|c|c|c|}
\hline \multicolumn{4}{|l|}{ Complex 1} \\
\hline $\mathrm{Cu}(1)-\mathrm{I}(1)$ & $2.5910(5)$ & $\mathrm{Cu}(1)-\mathrm{I}(1 \mathrm{~A})$ & $2.5973(5)$ \\
\hline $\mathrm{Cu}(1)-\mathrm{I}(1)$ & $2.5910(5)$ & $\mathrm{Cu}(1)-\mathrm{I}(1 \mathrm{~A})$ & $2.5973(5)$ \\
\hline $\mathrm{Cu}(1)-\mathrm{N}(4)$ & $2.077(3)$ & $\mathrm{Cu}(1)-\mathrm{N}(2)$ & $2.145(2)$ \\
\hline $\mathrm{Cu}(1) \cdots \mathrm{Cu}(1 \mathrm{~A})$ & $2.5870(7)$ & & \\
\hline $\mathrm{Cu}(1)-\mathrm{I}(1)-\mathrm{Cu}(1 \mathrm{~A})$ & $59.818(15)$ & $\mathrm{N}(4)-\mathrm{Cu}(1)-\mathrm{N}(2)$ & $78.99(10)$ \\
\hline $\mathrm{N}(4)-\mathrm{Cu}(1)-\mathrm{I}(1)$ & $114.80(7)$ & $\mathrm{N}(2)-\mathrm{Cu}(1)-\mathrm{I}(1)$ & $110.72(7)$ \\
\hline $\mathrm{N}(4)-\mathrm{Cu}(1)-\mathrm{I}(1 \mathrm{~A})$ & $106.70(7)$ & $\mathrm{N}(2)-\mathrm{Cu}(1)-\mathrm{I}(1 \mathrm{~A})$ & $118.16(7)$ \\
\hline $\mathrm{I}(1)-\mathrm{Cu}(1)-\mathrm{I}(1 \mathrm{~A})$ & $120.182(15)$ & & \\
\hline \multicolumn{4}{|l|}{ Complex 2} \\
\hline $\mathrm{Cu}(1)-\mathrm{I}(1)$ & $2.5872(18)$ & $\mathrm{Cu}(1)-\mathrm{I}(2)$ & $2.6111(18)$ \\
\hline $\mathrm{Cu}(1)-\mathrm{N}(3)$ & $2.095(10)$ & $\mathrm{Cu}(1)-\mathrm{N}(1)$ & $2.121(10)$ \\
\hline $\mathrm{Cu}(2)-\mathrm{I}(1)$ & $2.7068(17)$ & $\mathrm{Cu}(2)-\mathrm{I}(2)$ & $2.6861(18)$ \\
\hline $\mathrm{Cu}(2)-\mathrm{I}(2 \mathrm{~B})$ & $2.6481(18)$ & $\mathrm{Cu}(2)-\mathrm{I}(3)$ & $2.6421(17)$ \\
\hline $\mathrm{Cu}(3)-\mathrm{I}(1)$ & $2.6361(17)$ & $\mathrm{Cu}(3)-\mathrm{I}(3)$ & $2.6768(17)$ \\
\hline $\mathrm{Cu}(3)-\mathrm{I}(3 \mathrm{~A})$ & $2.6971(18)$ & $\mathrm{Cu}(3)-\mathrm{N}(4 \mathrm{~A})$ & $2.043(10)$ \\
\hline $\mathrm{Cu}(1) \cdots \mathrm{Cu}(2)$ & $2.630(2)$ & $\mathrm{Cu}(2) \cdots \mathrm{Cu}(3)$ & $2.891(2)$ \\
\hline $\mathrm{Cu}(2) \cdots \mathrm{Cu}(2 \mathrm{~B})$ & $2.975(3)$ & $\mathrm{Cu}(3) \cdots \mathrm{Cu}(3 \mathrm{~A})$ & $2.714(3)$ \\
\hline $\mathrm{N}(3)-\mathrm{Cu}(1)-\mathrm{N}(1)$ & $78.7(4)$ & $\mathrm{N}(3)-\mathrm{Cu}(1)-\mathrm{I}(1)$ & $106.9(3)$ \\
\hline $\mathrm{N}(1)-\mathrm{Cu}(1)-\mathrm{I}(1)$ & $124.7(3)$ & $\mathrm{N}(3)-\mathrm{Cu}(1)-\mathrm{I}(2)$ & $110.7(3)$ \\
\hline $\mathrm{N}(1)-\mathrm{Cu}(1)-\mathrm{I}(2)$ & $102.6(3)$ & $\mathrm{I}(1)-\mathrm{Cu}(1)-\mathrm{I}(2)$ & $123.90(6)$ \\
\hline $\mathrm{I}(3)-\mathrm{Cu}(2)-\mathrm{I}(2 \mathrm{~B})$ & $109.04(6)$ & $\mathrm{I}(3)-\mathrm{Cu}(2)-\mathrm{I}(2)$ & $104.50(6)$ \\
\hline $\mathrm{I}(2)-\mathrm{Cu}(2)-\mathrm{I}(2 \mathrm{~B})$ & $112.20(6)$ & $\mathrm{I}(3)-\mathrm{Cu}(2)-\mathrm{I}(1)$ & $112.20(6)$ \\
\hline $\mathrm{I}(2 \mathrm{~B})-\mathrm{Cu}(2)-\mathrm{I}(1)$ & $102.35(6)$ & $\mathrm{I}(2)-\mathrm{Cu}(2)-\mathrm{I}(1)$ & $116.57(6)$ \\
\hline $\mathrm{N}(4 \mathrm{~A})-\mathrm{Cu}(3)-\mathrm{I}(1)$ & $119.0(3)$ & $\mathrm{N}(4 \mathrm{~A})-\mathrm{Cu}(3)-\mathrm{I}(3)$ & $99.7(3)$ \\
\hline $\mathrm{I}(1)-\mathrm{Cu}(3)-\mathrm{I}(3)$ & $113.37(6)$ & $\mathrm{N}(4 \mathrm{~A})-\mathrm{Cu}(3)-\mathrm{I}(3 \mathrm{~A})$ & $105.4(3)$ \\
\hline $\mathrm{I}(1)-\mathrm{Cu}(3)-\mathrm{I}(3 \mathrm{~A})$ & $100.83(6)$ & $\mathrm{I}(3) \mathrm{Cu}(3) \mathrm{I}(3 \mathrm{~A})$ & $119.34(6)$ \\
\hline \multicolumn{4}{|l|}{ Compound 3} \\
\hline $\mathrm{Cu}(2)-\mathrm{I}(1)$ & $2.6076(13)$ & $\mathrm{Cu}(1)-\mathrm{I}(1)$ & $2.7053(14)$ \\
\hline $\mathrm{Cu}(2)-\mathrm{I}(2)$ & $2.5776(13)$ & $\mathrm{Cu}(1)-\mathrm{I}(2)$ & $2.6884(15)$ \\
\hline $\mathrm{Cu}(1)-\mathrm{N}(4)$ & $2.033(7)$ & $\mathrm{Cu}(1)-\mathrm{I}(1 \mathrm{~A})$ & $2.6402(14)$ \\
\hline $\mathrm{Cu}(2)-\mathrm{N}(3 \mathrm{~B})$ & $2.071(8)$ & $\mathrm{Cu}(2)-\mathrm{N}(1 \mathrm{~B})$ & $2.117(6)$ \\
\hline $\mathrm{Cu}(1) \cdots \mathrm{Cu}(2)$ & $2.6736(17)$ & & \\
\hline $\mathrm{N}(4)-\mathrm{Cu}(1)-\mathrm{I}(1 \mathrm{~A})$ & $108.7(2)$ & $\mathrm{N}(4)-\mathrm{Cu}(1)-\mathrm{I}(2)$ & $101.1(2)$ \\
\hline
\end{tabular}




$\begin{array}{llll}\mathrm{I}(1 \mathrm{~A})-\mathrm{Cu}(1)-\mathrm{I}(2) & 121.14(5) & \mathrm{N}(4)-\mathrm{Cu}(1)-\mathrm{I}(1) & 107.6(2) \\ \mathrm{I}(1 \mathrm{~A})-\mathrm{Cu}(1)-\mathrm{I}(1) & 107.36(5) & \mathrm{I}(2)-\mathrm{Cu}(1)-\mathrm{I}(1) & 110.10(5) \\ \mathrm{I}(2)-\mathrm{Cu}(2)-\mathrm{I}(1) & 116.99(5) & \mathrm{N}(3 \mathrm{~B})-\mathrm{Cu}(2)-\mathrm{N}(1 \mathrm{~B}) & 79.2(3) \\ \mathrm{N}(3 \mathrm{~B})-\mathrm{Cu}(2)-\mathrm{I}(2) & 111.0(2) & \mathrm{N}(1 \mathrm{~B})-\mathrm{Cu}(2)-\mathrm{I}(2) & 117.67(18) \\ \mathrm{N}(3 \mathrm{~B})-\mathrm{Cu}(2)-\mathrm{I}(1) & 115.33(19) & \mathrm{N}(1 \mathrm{~B})-\mathrm{Cu}(2)-\mathrm{I}(1) & 111.08(18)\end{array}$

Compound 4

$\mathrm{Cu}(1)-\mathrm{I}(1)$

2.553(3)

$\mathrm{Cu}(2)-\mathrm{I}(2)$

2.645(2)

$\mathrm{Cu}(1)-\mathrm{N}(3)$

$2.070(10)$

$\mathrm{Cu}(1)-\mathrm{N}(1)$

2.102(10)

$\mathrm{Cu}(1)-\mathrm{I}(1 \mathrm{~A})$

2.562(3)

$\mathrm{Cu}(2)-\mathrm{N}(4)$

2.046(10)

$\mathrm{Cu}(2)-\mathrm{I}(2 \mathrm{~B})$

2.652(3)

$\mathrm{Cu}(2)-\mathrm{I}(2 \mathrm{C})$

2.667(3)

$\mathrm{Cu}(2) \cdots \mathrm{Cu}(2 \mathrm{C})$

2.843(2)

$\mathrm{Cu}(2) \cdots \mathrm{Cu}(2 \mathrm{~B})$

2.843(2)

$\mathrm{N}(3)-\mathrm{Cu}(1)-\mathrm{N}(1)$

78.9(4)

$\mathrm{N}(3)-\mathrm{Cu}(1)-\mathrm{I}(1)$

107.0(4)

$\mathrm{N}(1)-\mathrm{Cu}(1)-\mathrm{I}(1)$

132.4(4)

$\mathrm{N}(3)-\mathrm{Cu}(1)-\mathrm{I}(1 \mathrm{~A})$

124.6(4)

$\mathrm{N}(1)-\mathrm{Cu}(1)-\mathrm{I}(1 \mathrm{~A})$

107.6(4)

$\mathrm{N}(4)-\mathrm{Cu}(2)-\mathrm{I}(2)$

105.7(3)

$\mathrm{I}(1)-\mathrm{Cu}(1)-\mathrm{I}(1 \mathrm{~A})$

106.48(7)

$\mathrm{I}(2)-\mathrm{Cu}(2)-\mathrm{I}(2 \mathrm{~B})$

115.43(9)

$\mathrm{N}(4)-\mathrm{Cu}(2)-\mathrm{I}(2 \mathrm{~B})$

113.5(4)

$\mathrm{I}(2)-\mathrm{Cu}(2)-\mathrm{I}(2 \mathrm{C})$

114.94(9)

106.3(4)

$\mathrm{I}(2 \mathrm{~B})-\mathrm{Cu}(2)-\mathrm{I}(2 \mathrm{C})$

100.77(7)

\section{Compound 5}

$\mathrm{Cu}(1)-\mathrm{I}(1)$

2.5874(9)

$\mathrm{Cu}(1)-\mathrm{N}(3)$

2.056(5)

$\mathrm{Cu}(1)-\mathrm{N}(2)$

2.157(5)

$\mathrm{Cu}(1)-\mathrm{I}(3 \mathrm{~A})$

2.5917(9)

$\mathrm{Cu}(2)-\mathrm{I}(1)$

2.7184(9)

$\mathrm{Cu}(2)-\mathrm{I}(2)$

2.6296(10)

$\mathrm{Cu}(2)-\mathrm{I}(3)$

2.6318(9)

$\mathrm{Cu}(2)-\mathrm{I}(3 \mathrm{~A})$

2.6837(9)

$\mathrm{Cu}(3)-\mathrm{N}(4 \mathrm{~B})$

$2.040(5)$

$\mathrm{Cu}(3)-\mathrm{I}(2)$

2.6691(9)

$\mathrm{Cu}(3)-\mathrm{I}(1)$

2.6373(9)

$\mathrm{Cu}(3)-\mathrm{I}(2 \mathrm{~B})$

2.6901(9)

$\mathrm{Cu}(1) \cdots \mathrm{Cu}(2)$

2.6176(11)

$\mathrm{Cu}(3) \cdots \mathrm{Cu}(3 \mathrm{~B})$

2.6781(17)

$\mathrm{Cu}(2) \cdots \mathrm{Cu}(3)$

2.9149(11)

$\mathrm{Cu}(2) \cdots \mathrm{Cu}(2 \mathrm{~A})$

2.9458(15)

$\mathrm{N}(3)-\mathrm{Cu}(1)-\mathrm{N}(2)$

$79.0(2)$

$\mathrm{N}(2)-\mathrm{Cu}(1)-\mathrm{I}(1)$

123.33(12)

$\mathrm{N}(3)-\mathrm{Cu}(1)-\mathrm{I}(1)$

107.10(15)

$\mathrm{N}(3)-\mathrm{Cu}(1)-\mathrm{I}(3 \mathrm{~A})$

111.31(13)

$\mathrm{N}(2)-\mathrm{Cu}(1)-\mathrm{I}(3 \mathrm{~A})$

102.02(13)

$\mathrm{I}(1)-\mathrm{Cu}(1)-\mathrm{I}(3 \mathrm{~A})$

124.78(3)

$\mathrm{I}(2)-\mathrm{Cu}(2)-\mathrm{I}(3)$

109.60(3)

$\mathrm{I}(2)-\mathrm{Cu}(2)-\mathrm{I}(3 \mathrm{~A})$

104.55(3)

$\mathrm{I}(3)-\mathrm{Cu}(2)-\mathrm{I}(3 \mathrm{~A})$

112.70(3)

$\mathrm{I}(3)-\mathrm{Cu}(2)-\mathrm{I}(1)$

102.44(3)

$\mathrm{I}(2)-\mathrm{Cu}(2)-\mathrm{I}(1)$

111.30(3)

$\mathrm{I}(3 \mathrm{~A})-\mathrm{Cu}(2)-\mathrm{I}(1)$

116.33(3)

$\mathrm{N}(4 \mathrm{~B})-\mathrm{Cu}(3)-\mathrm{I}(1)$

119.40(15)

$\mathrm{I}(1)-\mathrm{Cu}(3)-\mathrm{I}(2)$

112.63(3)

$\mathrm{N}(4 \mathrm{~B})-\mathrm{Cu}(3)-\mathrm{I}(2)$

99.83(13)

$\mathrm{N}(4 \mathrm{~B})-\mathrm{Cu}(3)-\mathrm{I}(2 \mathrm{~B})$

105.69(13)

$\mathrm{I}(1)-\mathrm{Cu}(3)-\mathrm{I}(2 \mathrm{~B})$

100.23(3)

$\mathrm{I}(2)-\mathrm{Cu}(3)-\mathrm{I}(2 \mathrm{~B})$

120.04(3)

Compound 6 


$\begin{array}{llll}\mathrm{Cu}(1)-\mathrm{I}(1) & 2.5740(8) & \mathrm{Cu}(1)-\mathrm{N}(4 \mathrm{~A}) & 2.032(4) \\ \mathrm{Cu}(1)-\mathrm{N}(3) & 2.056(4) & \mathrm{Cu}(1)-\mathrm{N}(2) & 2.157(4) \\ \mathrm{N}(4 \mathrm{~A})-\mathrm{Cu}(1)-\mathrm{N}(3) & 115.62(16) & \mathrm{N}(4 \mathrm{~A})-\mathrm{Cu}(1)-\mathrm{N}(2) & 128.55(17) \\ \mathrm{N}(3)-\mathrm{Cu}(1)-\mathrm{N}(2) & 78.88(16) & \mathrm{N}(4 \mathrm{~A})-\mathrm{Cu}(1)-\mathrm{I}(1) & 101.91(12) \\ \mathrm{N}(3)-\mathrm{Cu}(1)-\mathrm{I}(1) & 126.90(13) & \mathrm{N}(2)-\mathrm{Cu}(1)-\mathrm{I}(1) 106.31(11)\end{array}$

Complex 11

$\mathrm{Cu}(1)-\mathrm{I}(1)$

$2.5538(8)$

$\mathrm{Cu}(1)-\mathrm{N}(1)$

$1.967(3)$

$\mathrm{N}(1)-\mathrm{Cu}(1)-\mathrm{N}(1 \mathrm{~A})$

$131.15(19)$

$\mathrm{N}(1)-\mathrm{Cu}(1)-\mathrm{I}(1)$

$114.43(9)$

$\mathrm{N}(1 \mathrm{~A})-\mathrm{Cu}(1)-\mathrm{I}(1)$

$114.43(9)$

Symmetry codes: (A) $1+\mathrm{x}, 1-\mathrm{y}, 1 / 2+\mathrm{z}$ for $\mathbf{1}$. (A) $1-\mathrm{x},-\mathrm{y}, 2-\mathrm{z}$ and (B) $1-\mathrm{x}, 1-\mathrm{y}, 2-\mathrm{z}$ for 2 . (A) $1-\mathrm{x},-\mathrm{y}, 2-\mathrm{z}$; (B) $-1 / 2+\mathrm{x}, 1 / 2-\mathrm{y}, 1 / 2+\mathrm{z}$ for 3 . (A) $\mathrm{x},-1 / 2+\mathrm{y},-\mathrm{z}$; (B) $2-\mathrm{x},-1 / 2+\mathrm{y}, 1-\mathrm{z}$; (C) $2-\mathrm{x}, 1 / 2+\mathrm{y}, 1-\mathrm{z}$ for 4 . (A) $1-\mathrm{x}, 1-\mathrm{y}, 1-\mathrm{z}$ and (B) $1-\mathrm{x}, 2-\mathrm{y}, 1-\mathrm{z}$ for 5. (A) $2-\mathrm{x}, 1-\mathrm{y}$, $\mathrm{z}$ for 6. (A) $3 / 2+x,-1 / 2+y,-1 / 2-z$ for $\mathbf{1 1}$.

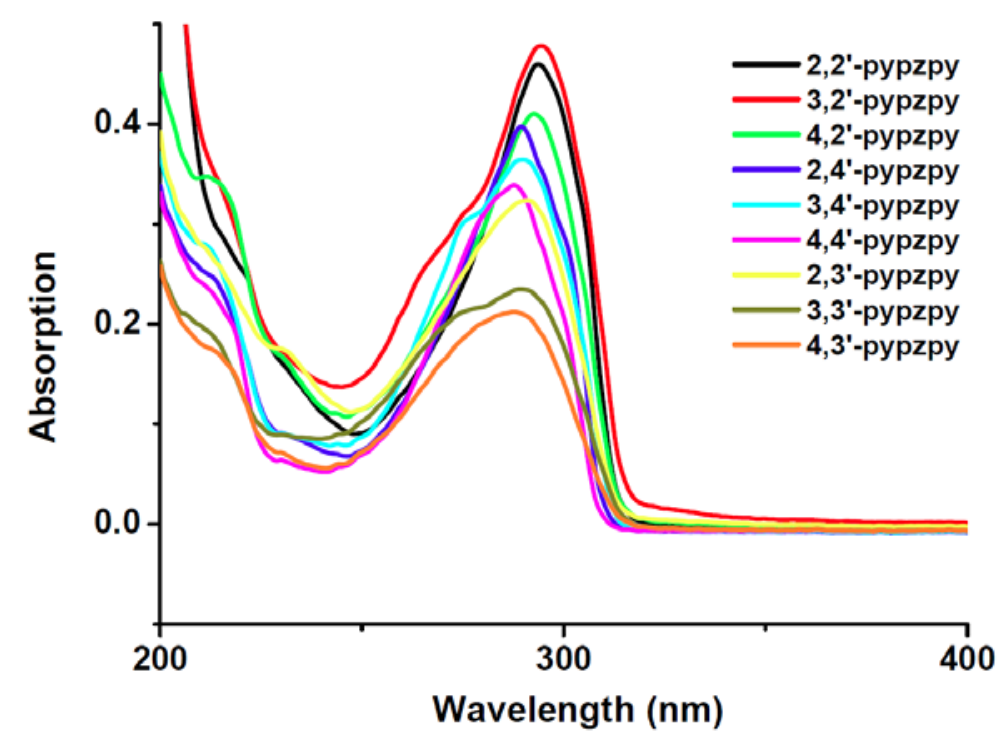

Figure S1. The UV-vis spectra of pypzpy ligands $\left(1 \times 10^{-5} \mathrm{~mol} / \mathrm{L}\right)$ in $\mathrm{MeCN}$ in a 1 -cm-thick glass cell. 


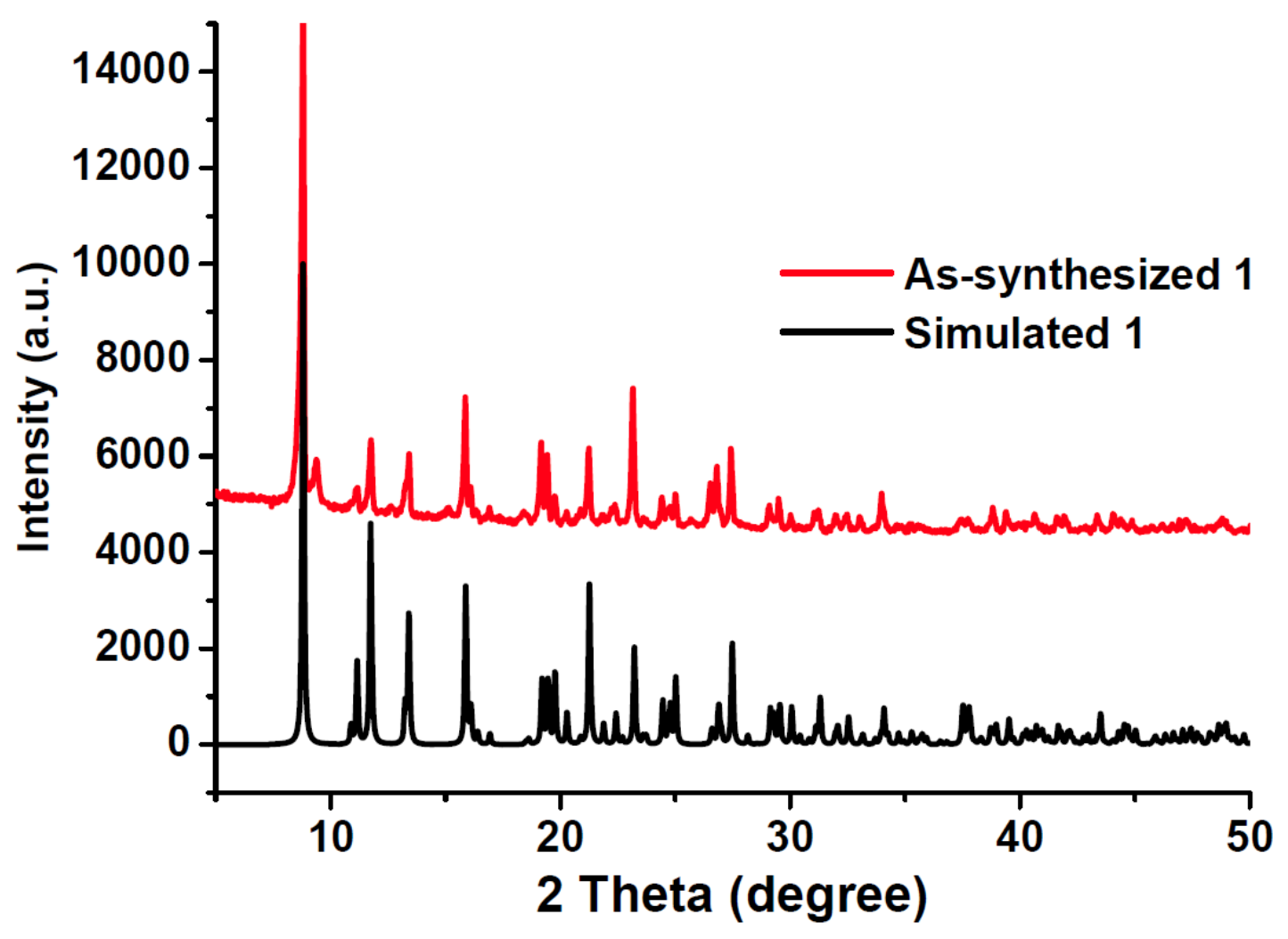

Figure S2. PXRD patterns for 1

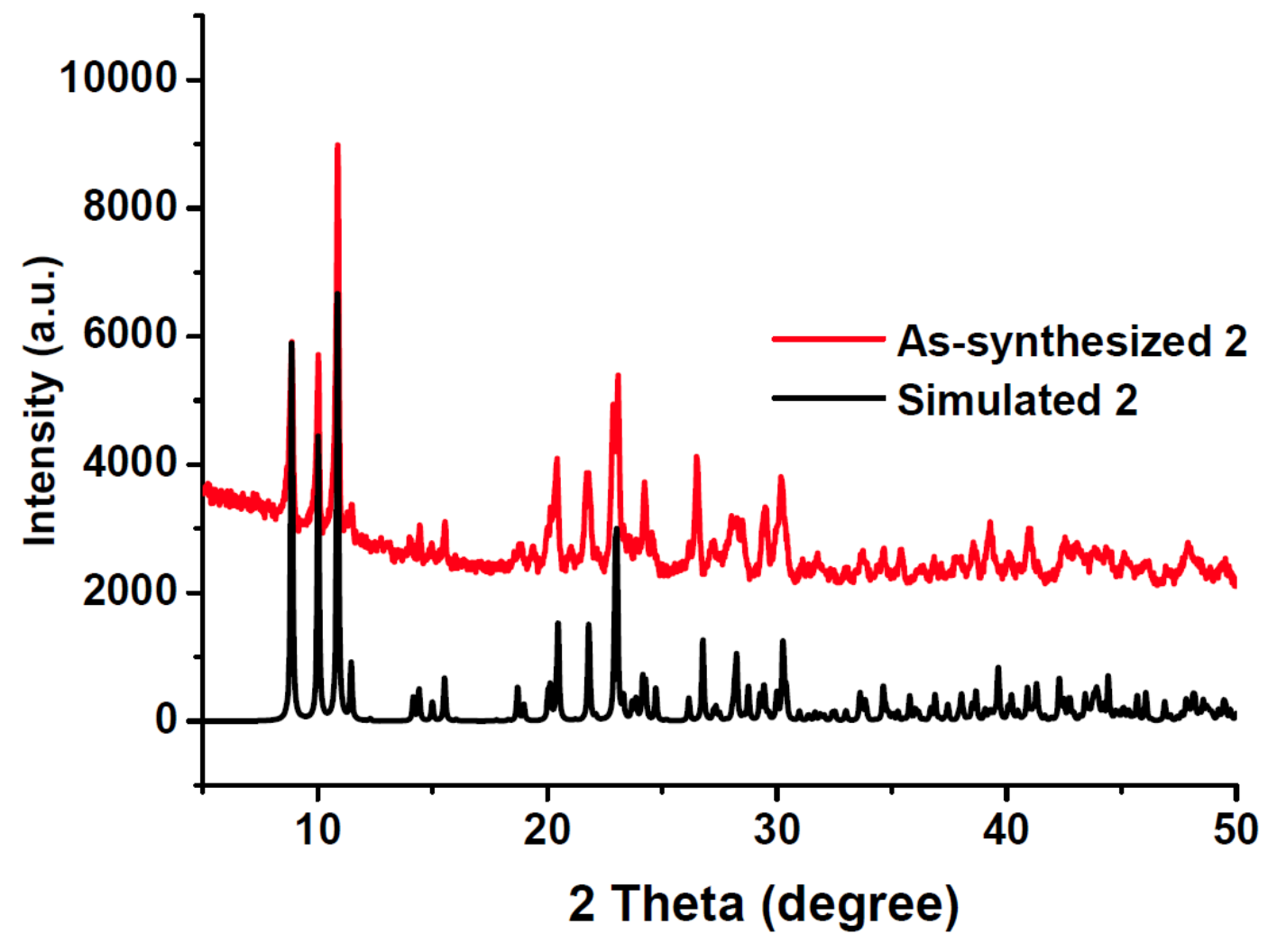

Figure S3. PXRD patterns for 2 


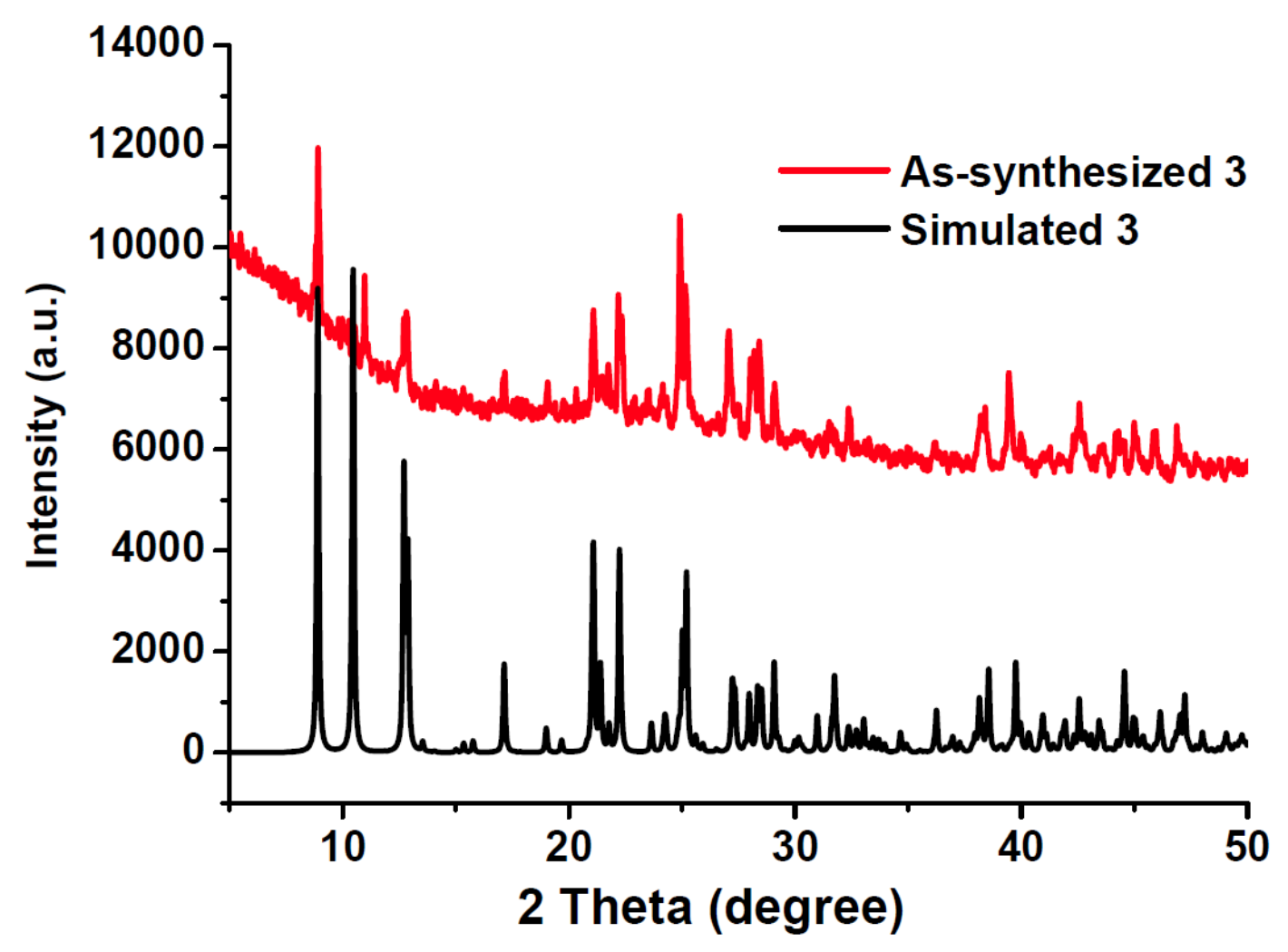

Figure S4. PXRD patterns for 3

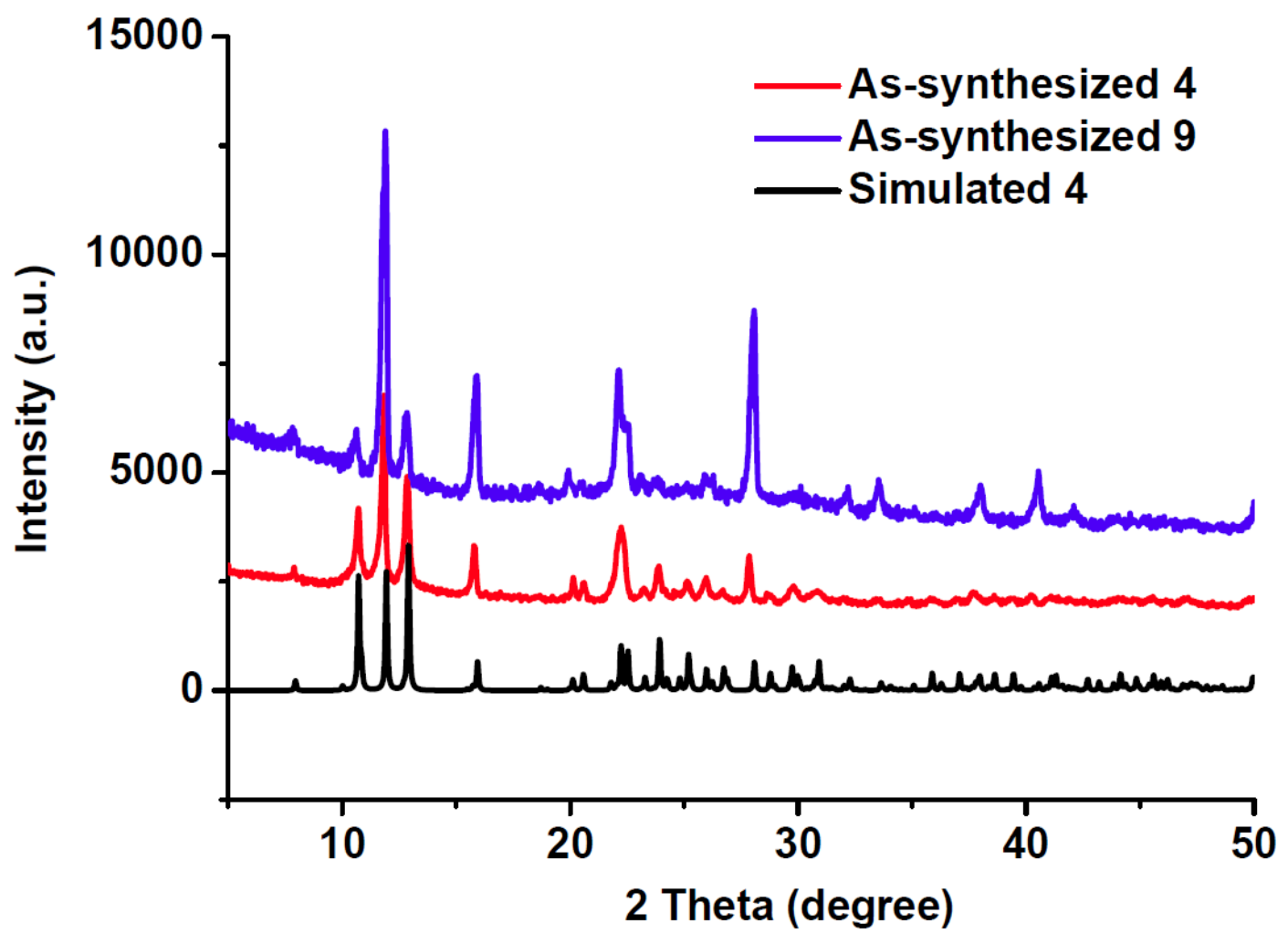

Figure S5. PXRD patterns for 4 and 9 


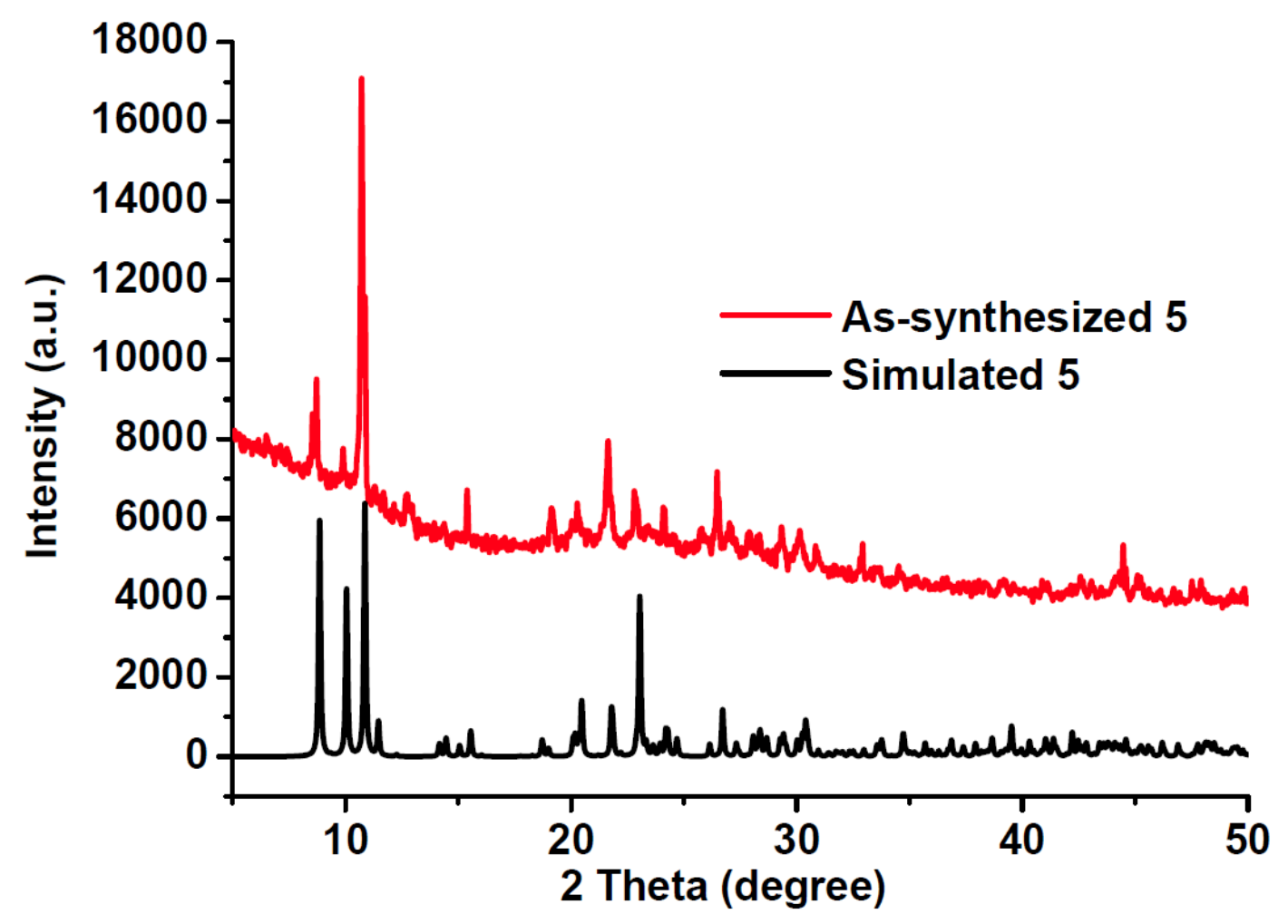

Figure S6. PXRD patterns for 5

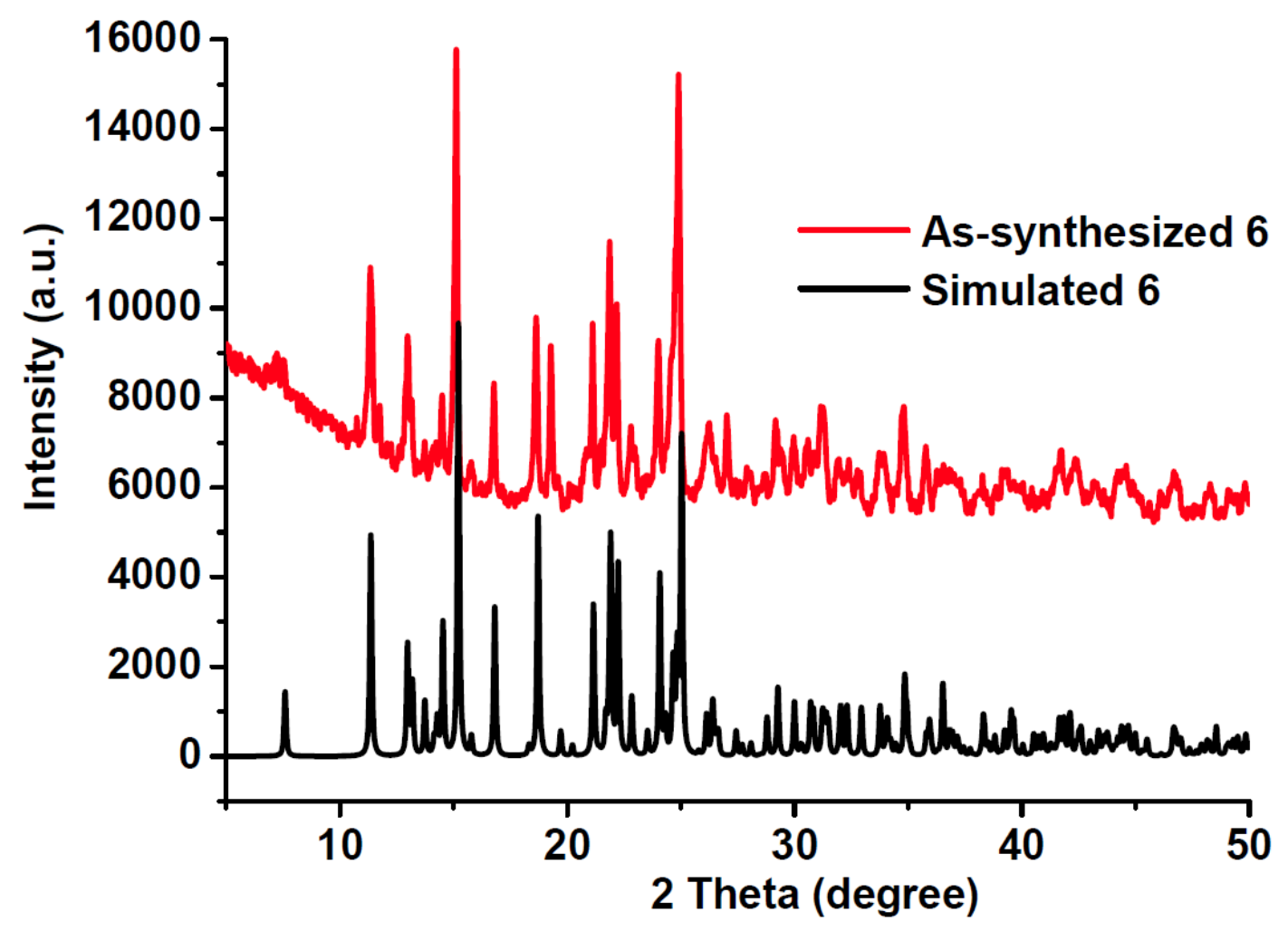

Figure S7. PXRD patterns for 6 


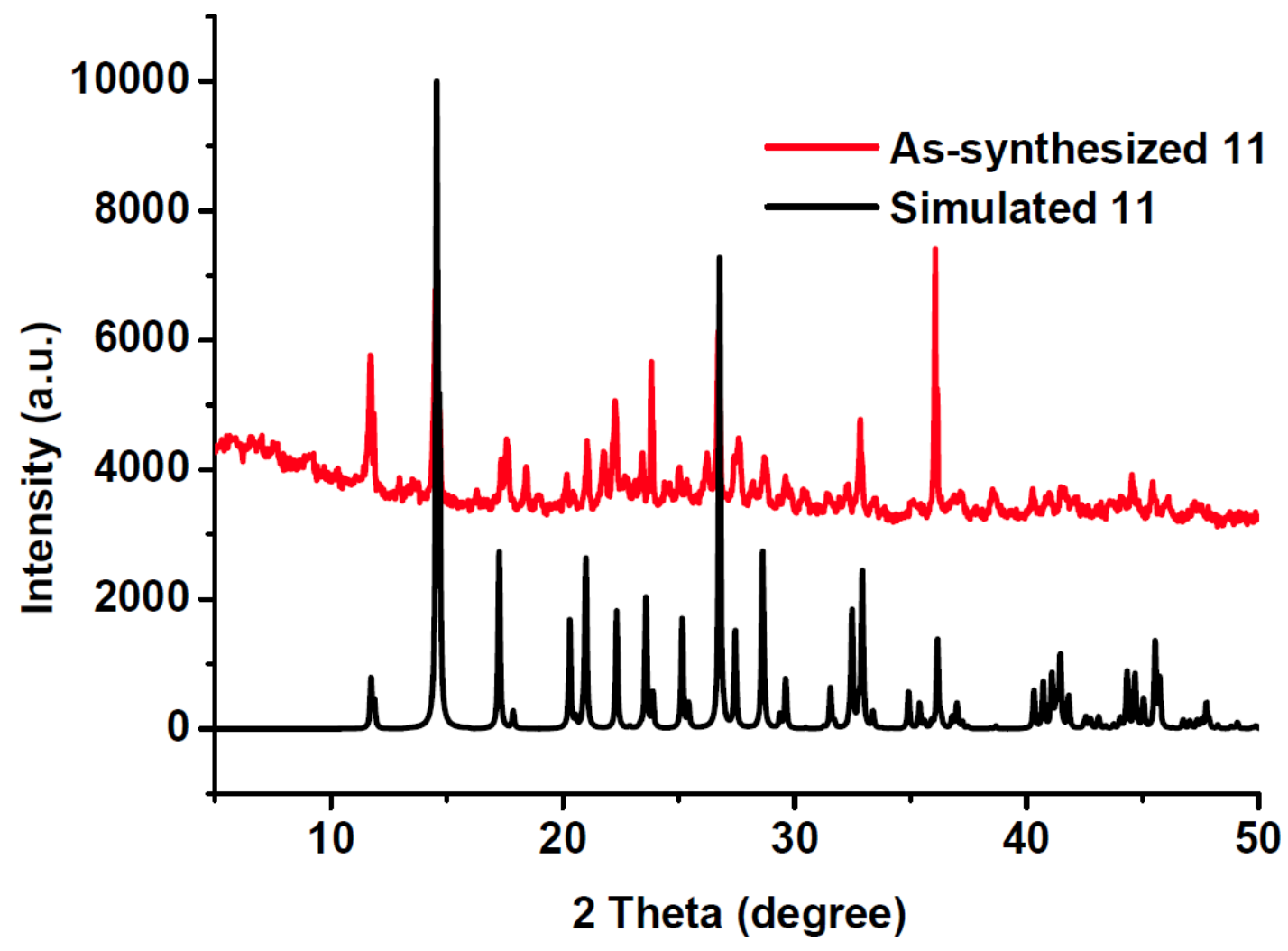

Figure S8. PXRD patterns for 11

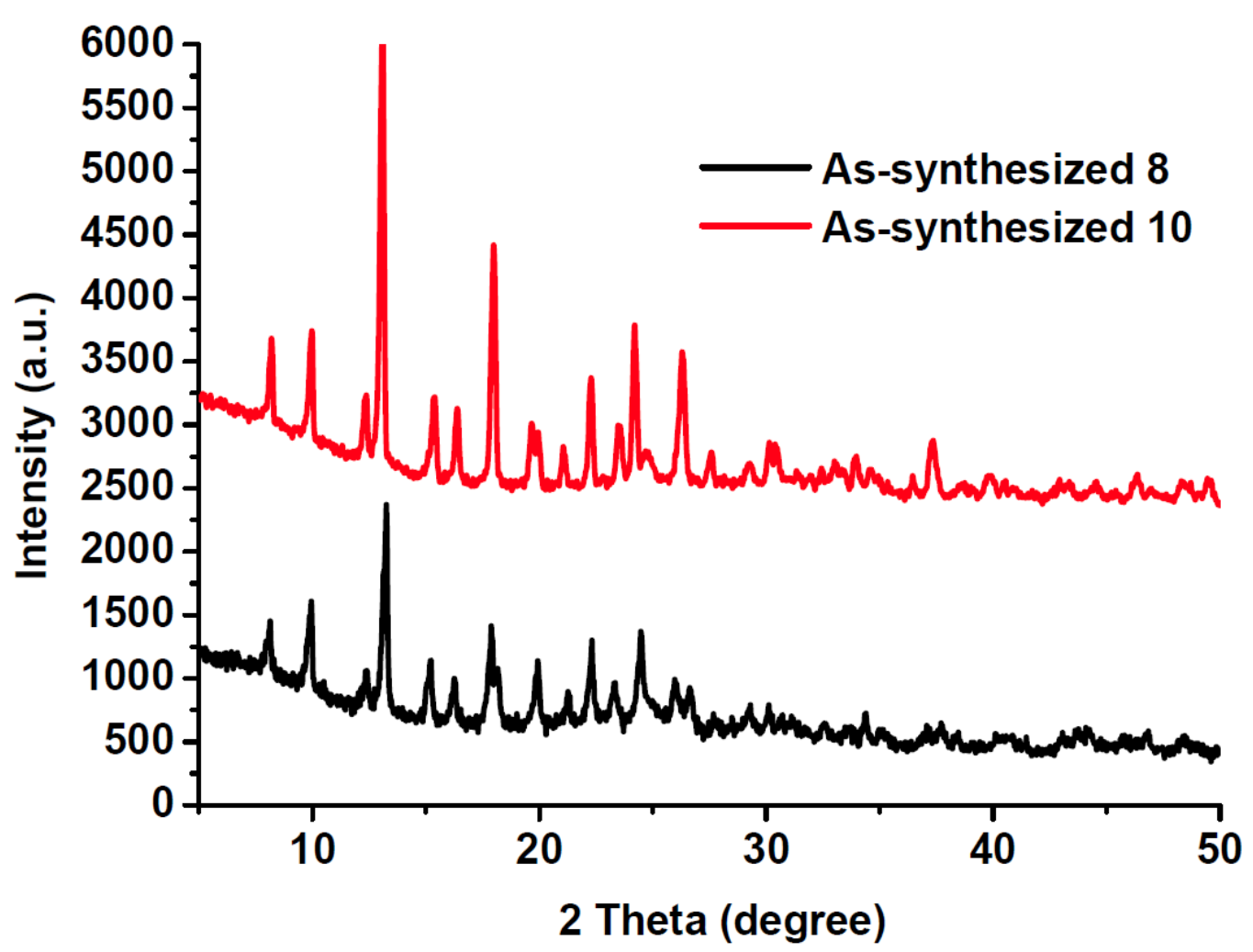

Figure S9. PXRD patterns for $\mathbf{8}$ and $\mathbf{1 0}$ 


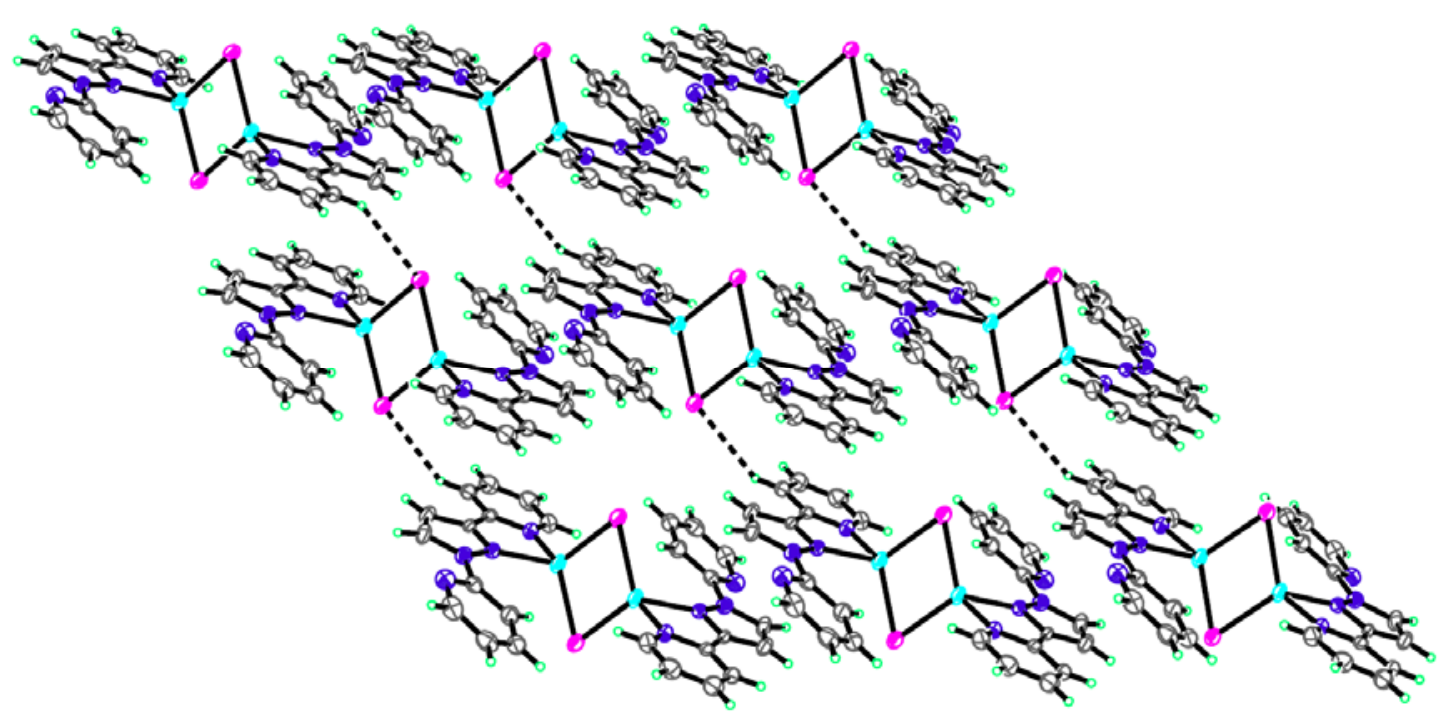

Figure S10. View of the 2D hydrogen-bound structure of $\mathbf{1}$.

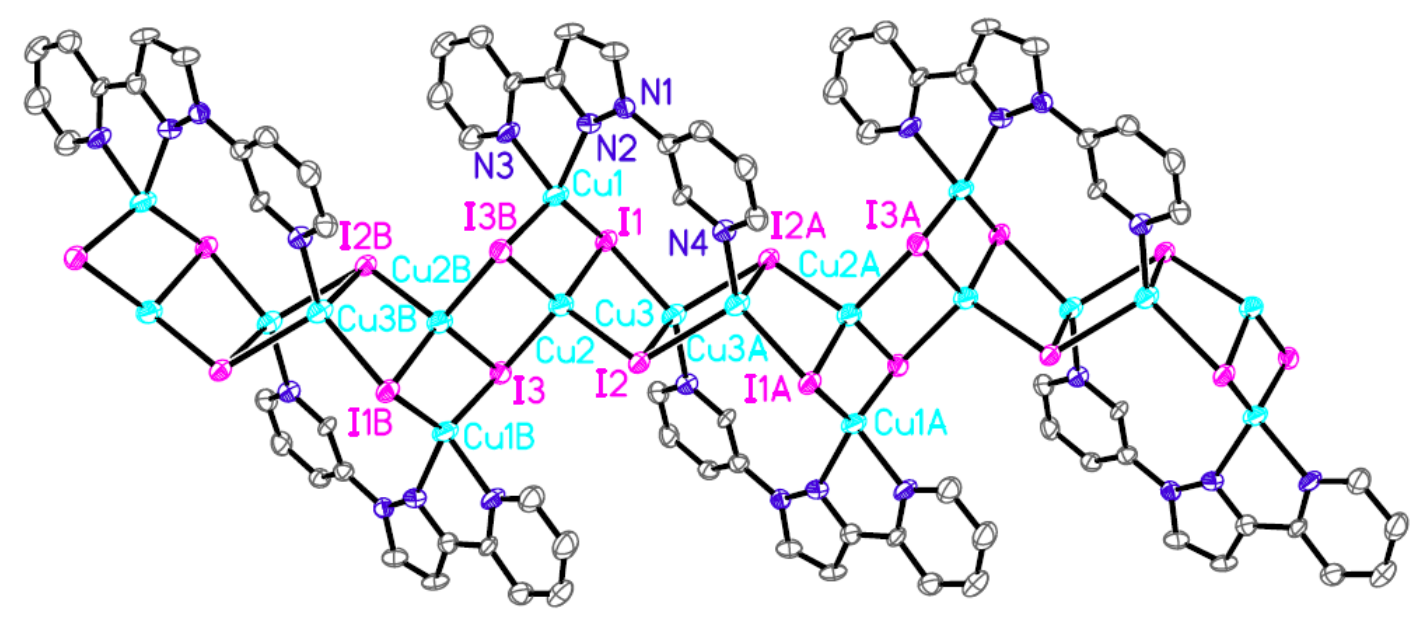

Figure S11. View of the molecular structure of 5 with a labeling scheme and $50 \%$ thermal ellipsoids. All $\mathrm{H}$ atoms were omitted for clarity. 


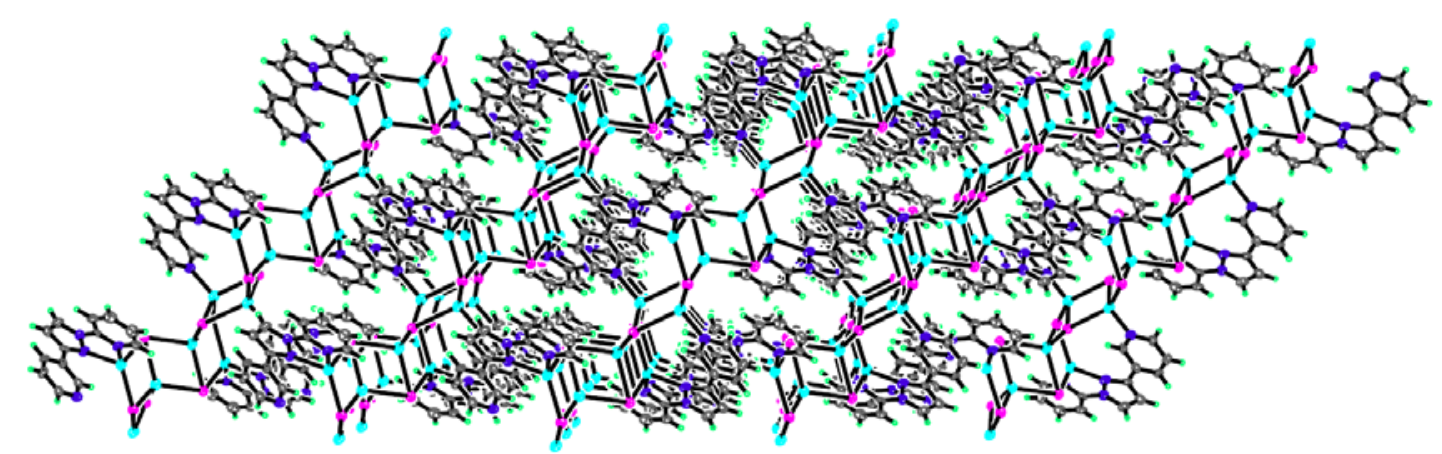

Figure S12. $\pi-\pi$ interactions between the two pyrazolyl rings and the two pyridyl rings of 2 .

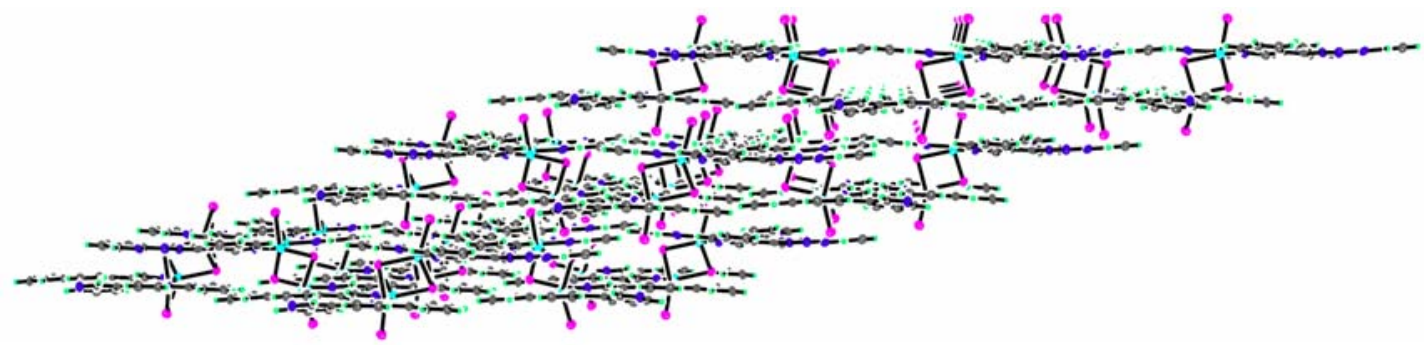

Figure S13. $\pi-\pi$ interactions between the two pyridyl rings of 3 .

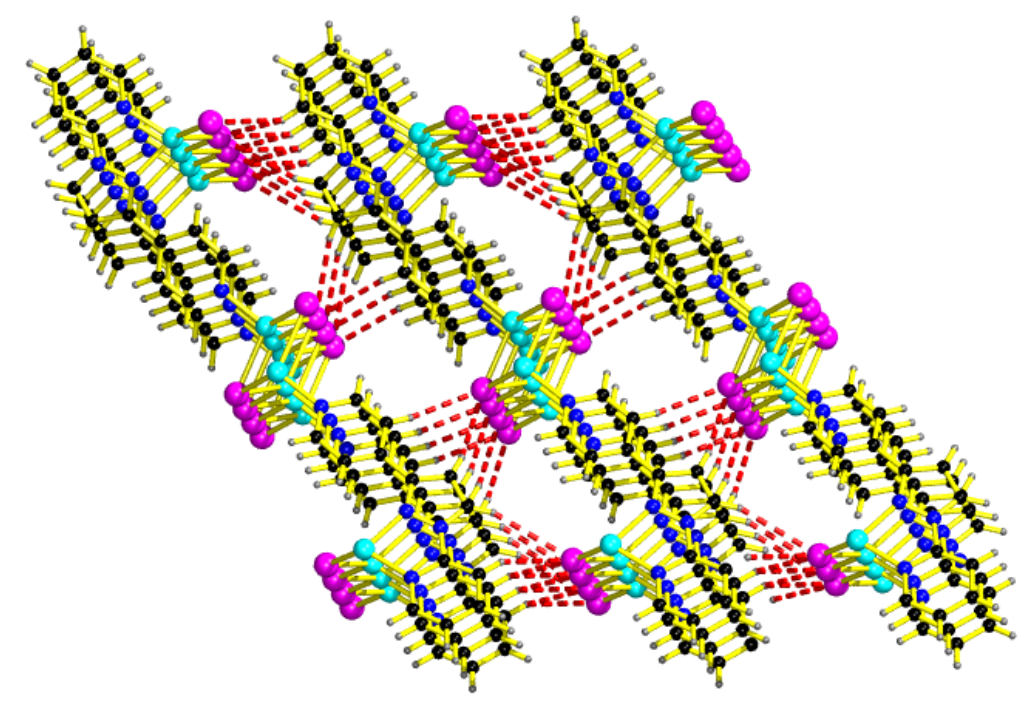

Figure S14. View of the 3D hydrogen-bound structure of 4. 


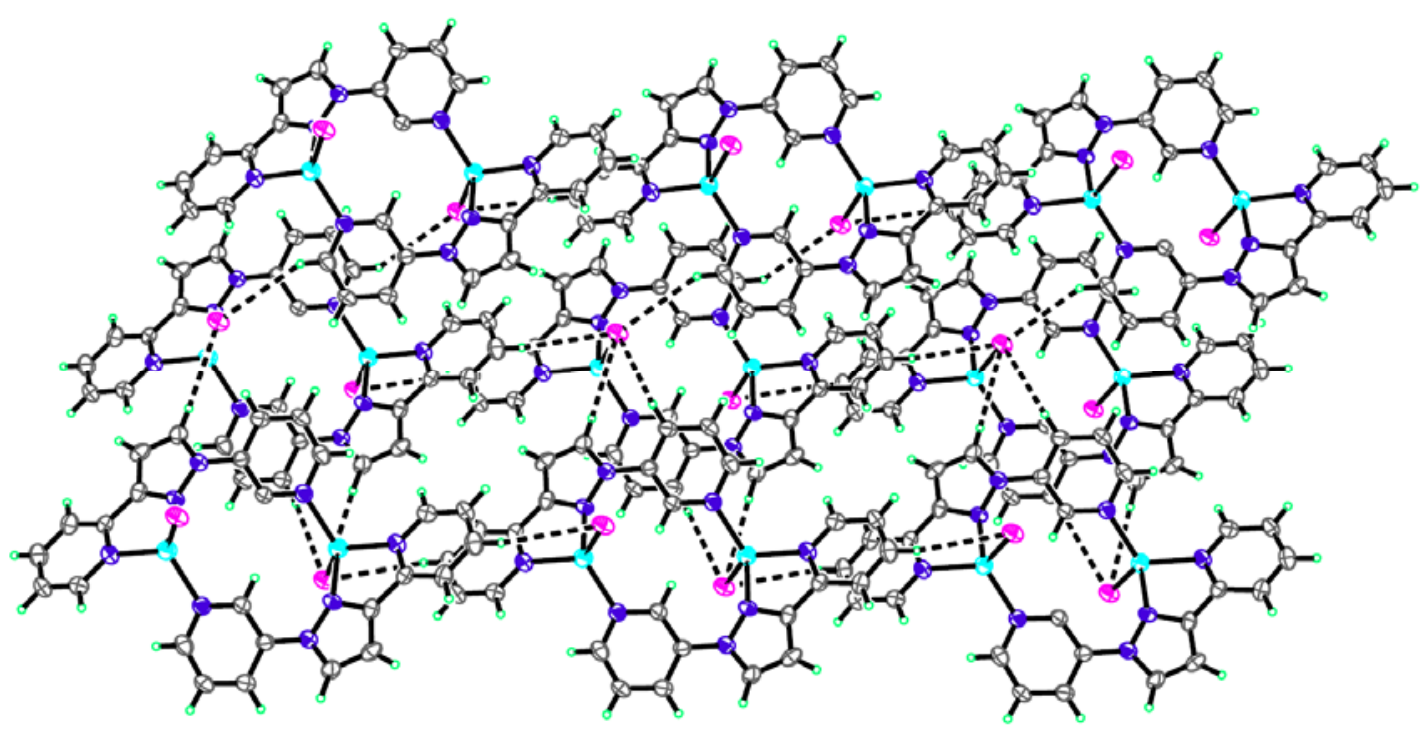

Figure S15. View of the 3D hydrogen-bound structure of 6 .

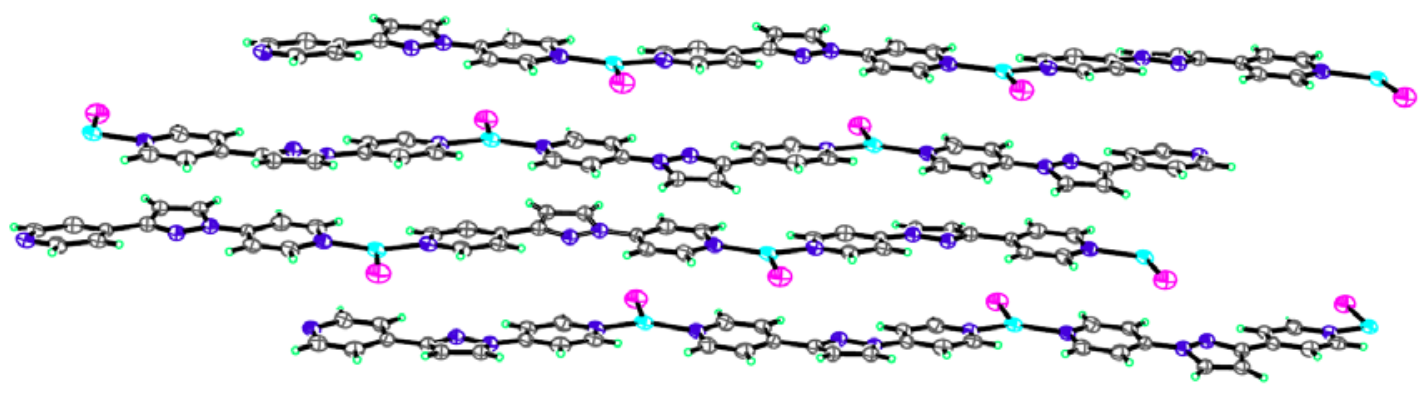

Figure S16. $\pi-\pi$ interactions between the pyridyl ring and pyrazolyl ring of 11 .

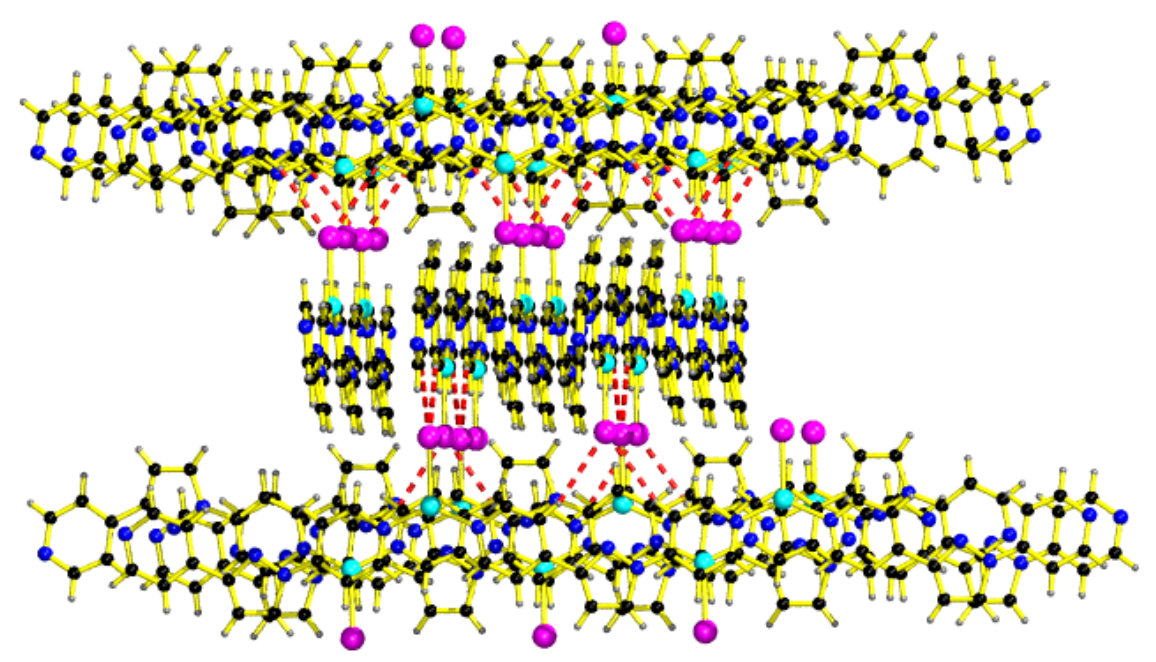

Figure S17. View of the 3D hydrogen-bound structure of 11. 


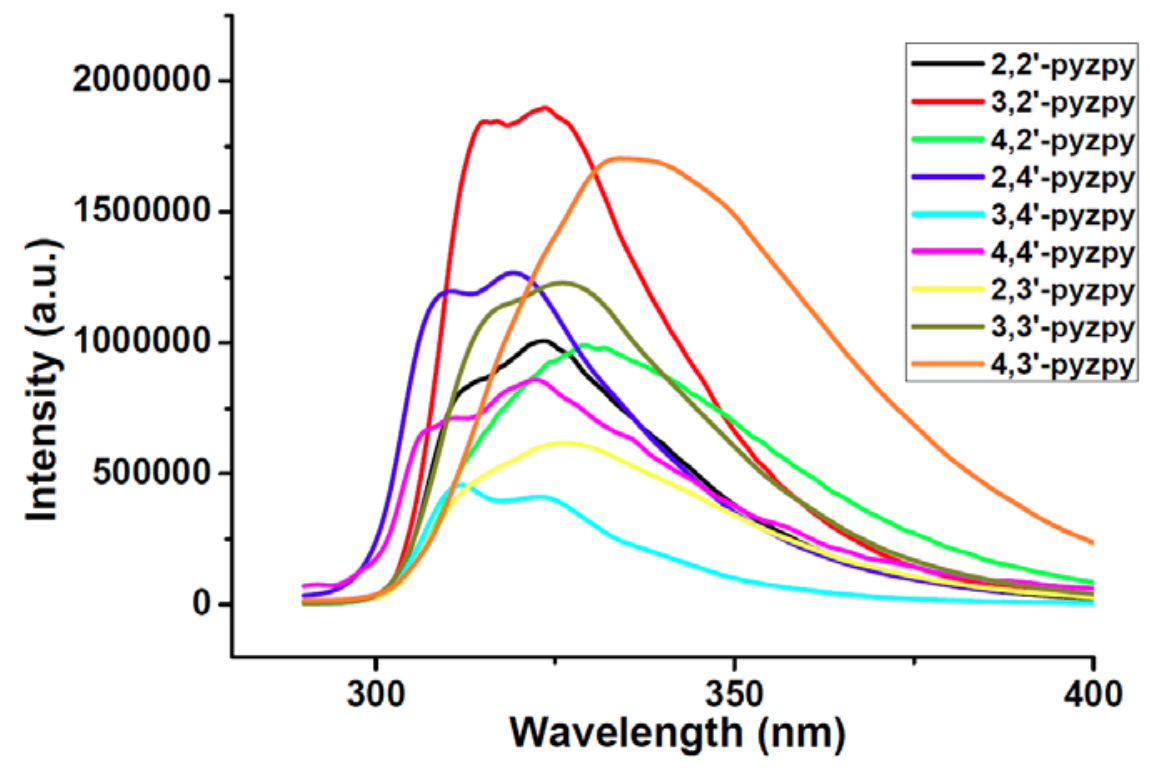

Figure S18. Emission spectra of pypzpy ligands in $\mathrm{MeCN}$ solution at room temperature. 

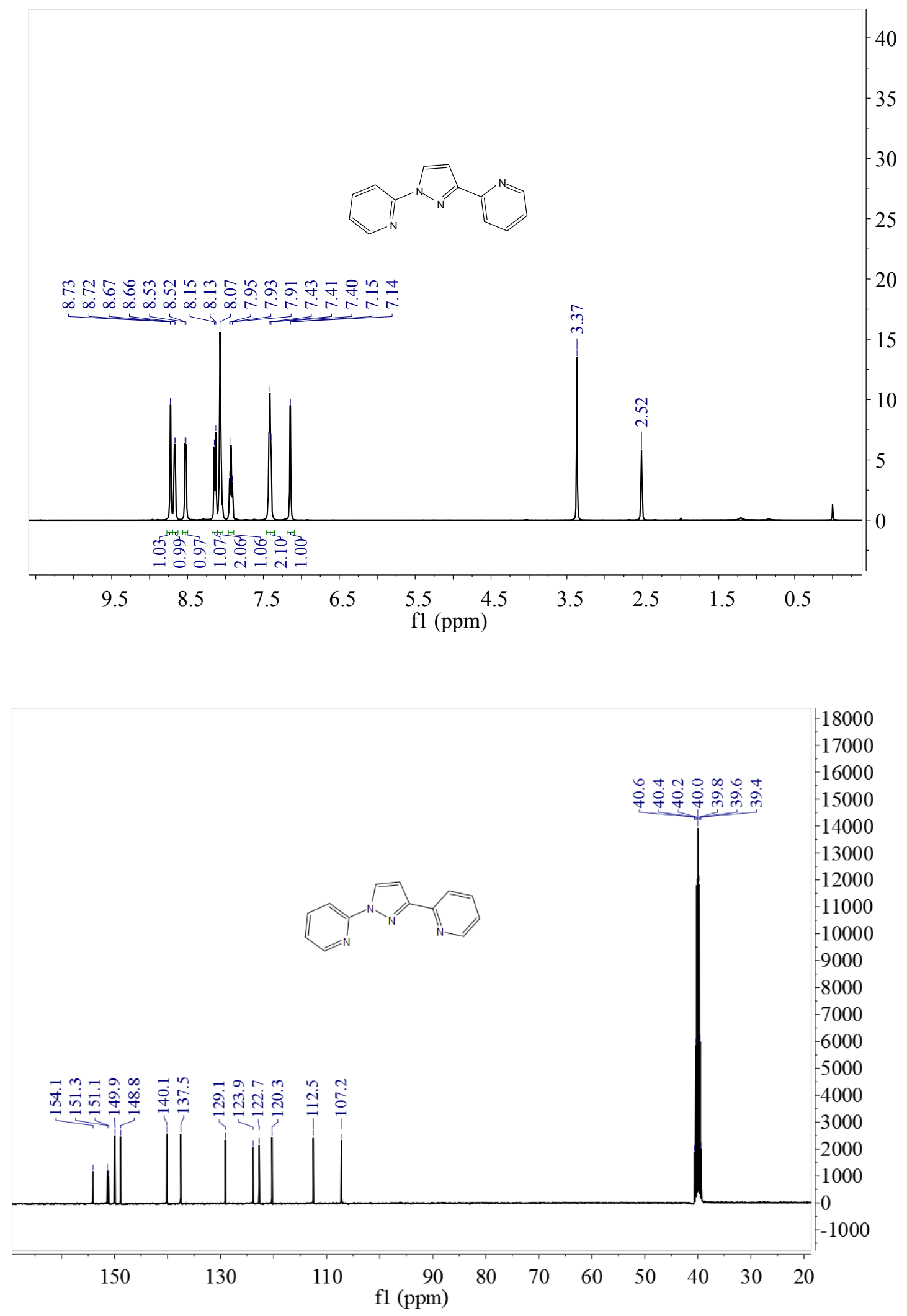

Figure S19. The ${ }^{1} \mathrm{H}$ and ${ }^{13} \mathrm{C}$ NMR spectra of 2,2'-pypzpy ligand. 

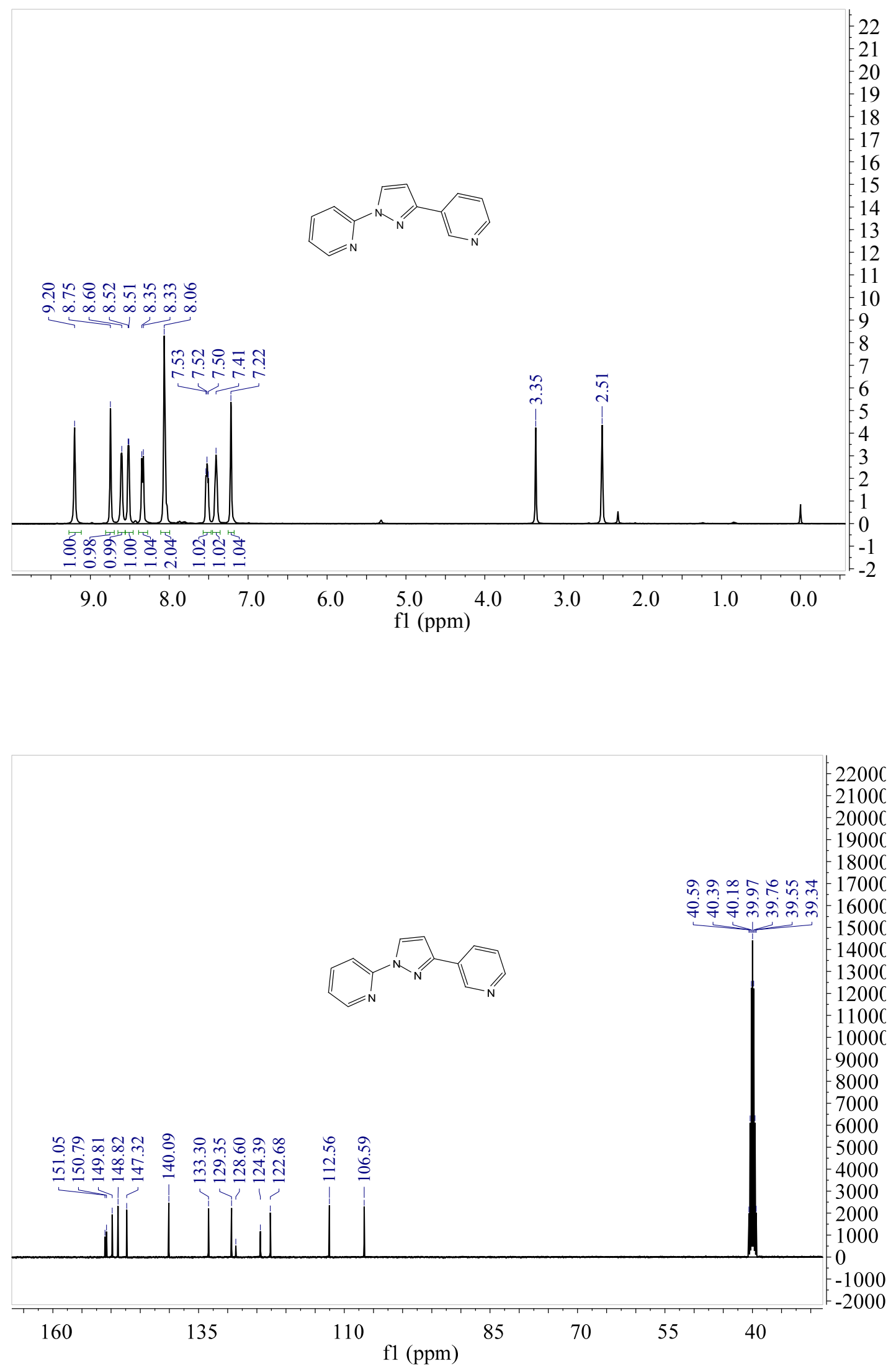

Figure S20. The ${ }^{1} \mathrm{H}$ and ${ }^{13} \mathrm{C}$ NMR spectra of 3,2'-pypzpy ligand. 

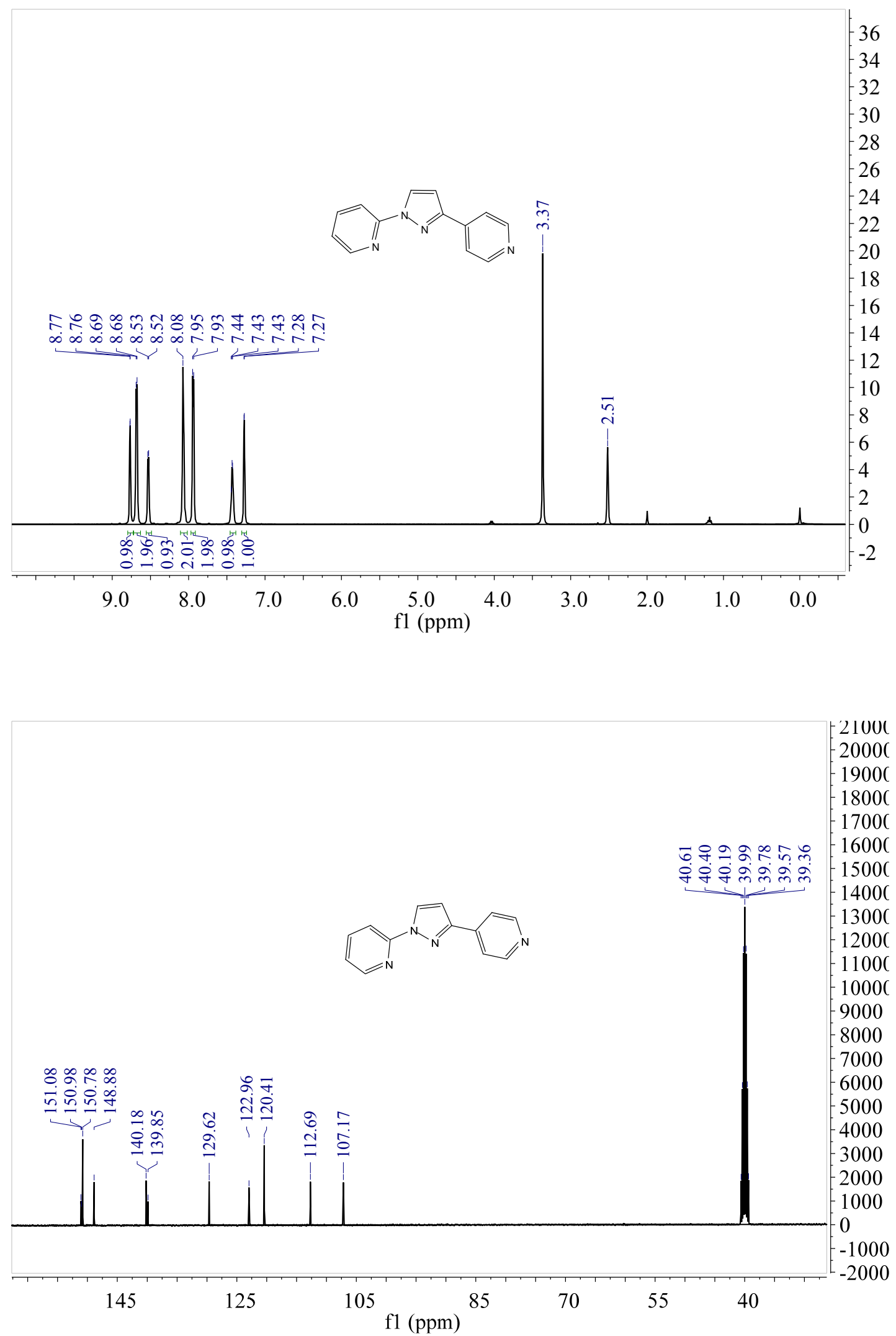

Figure S21. The ${ }^{1} \mathrm{H}$ and ${ }^{13} \mathrm{C}$ NMR spectra of 4,2'-pypzpy ligand. 

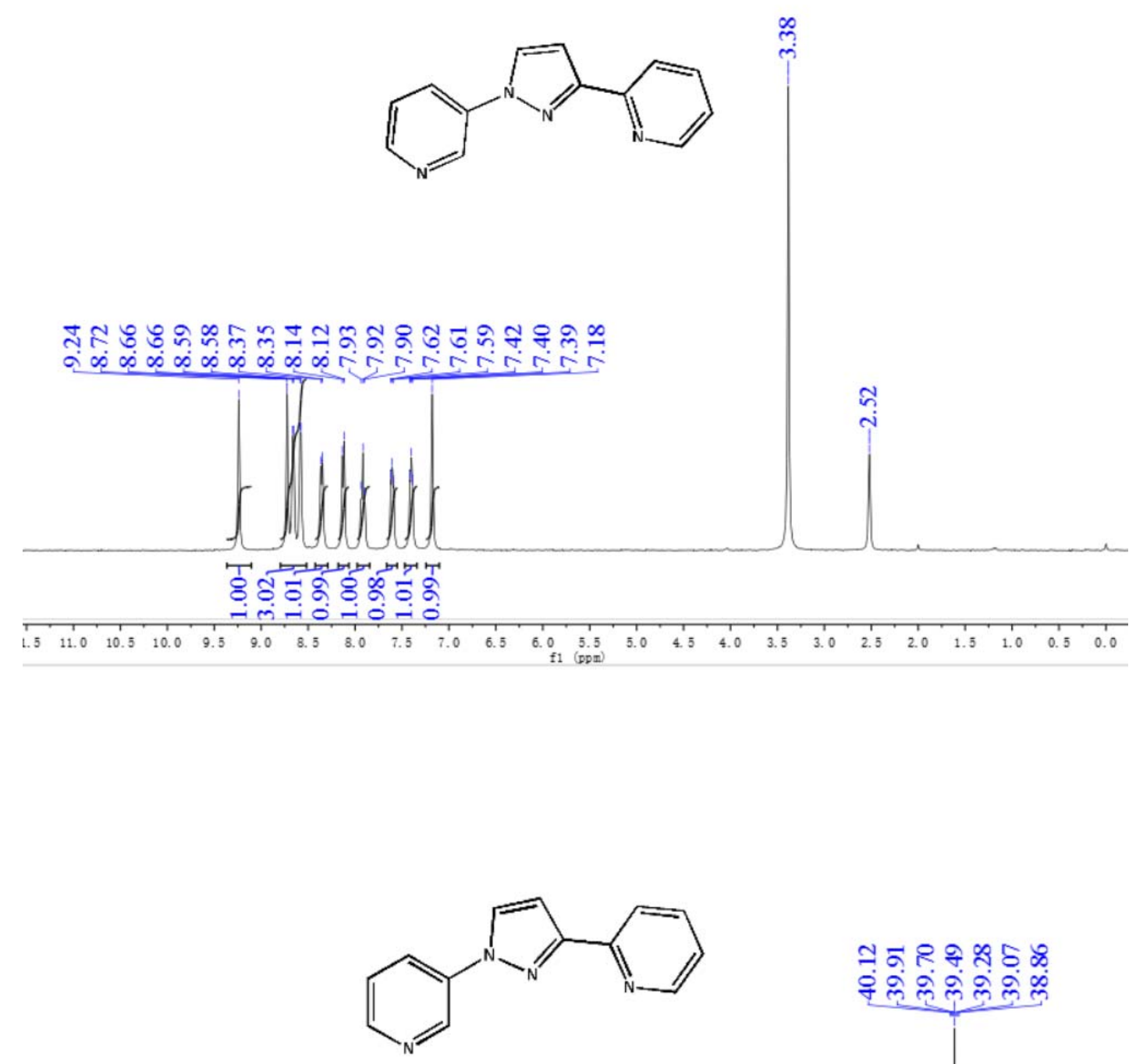

ติ

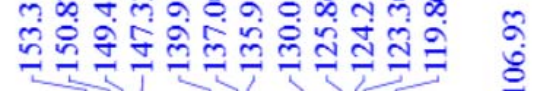

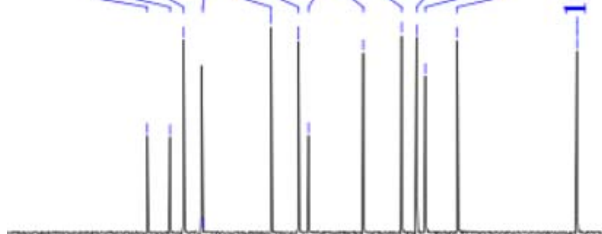

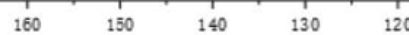

110

100

60

Figure S22. The ${ }^{1} \mathrm{H}$ and ${ }^{13} \mathrm{C}$ NMR spectra of 2,3'-pypzpy ligand. 

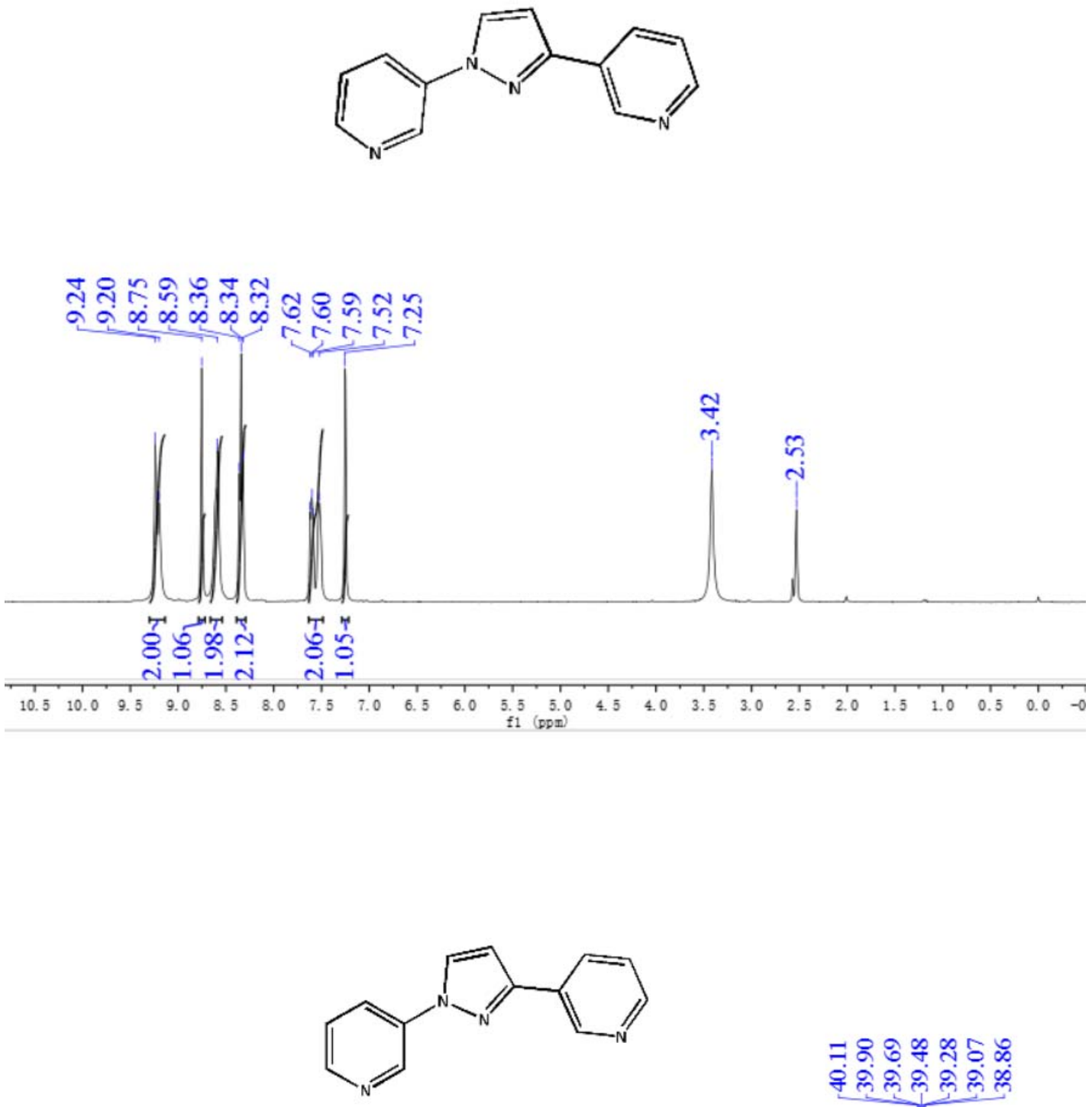

=

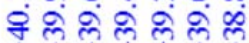

ชิ สุก

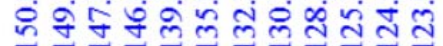

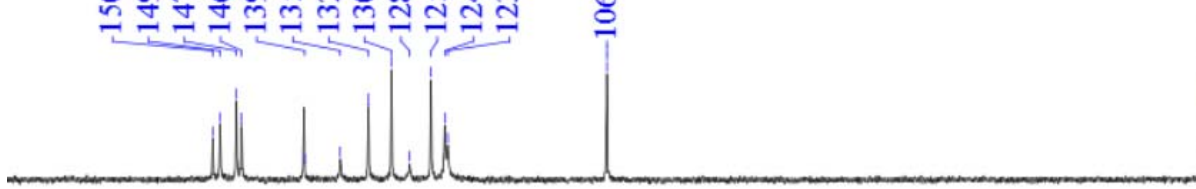

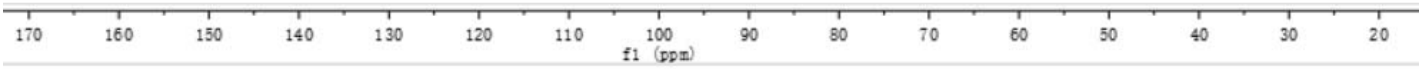

Figure S23. The ${ }^{1} \mathrm{H}$ and ${ }^{13} \mathrm{C}$ NMR spectra of 3,3'-pypzpy ligand. 


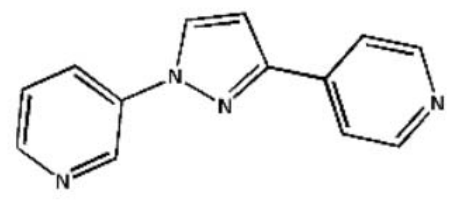

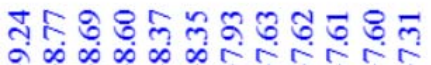
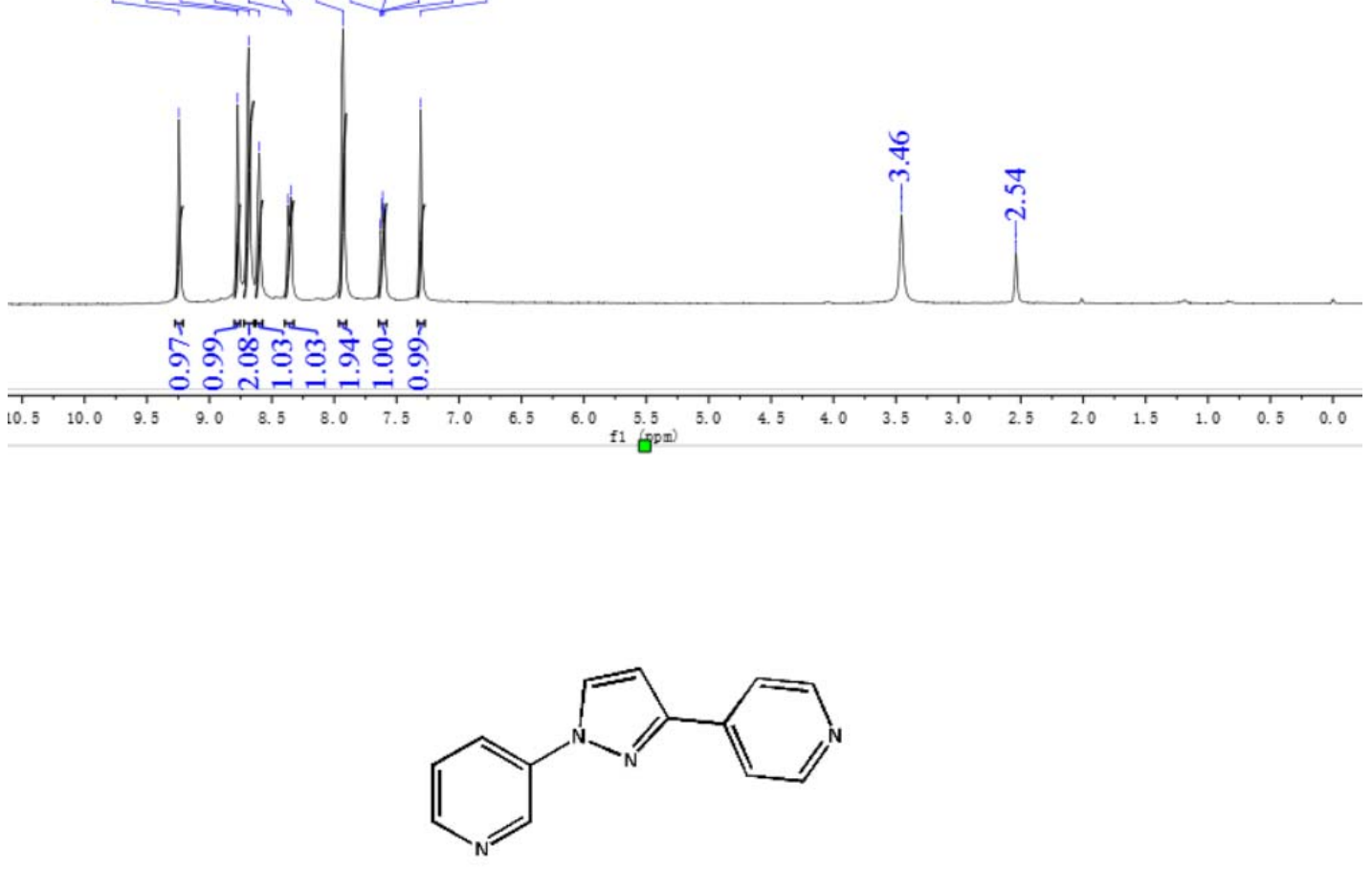

กิส ชุชุ

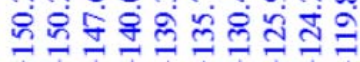

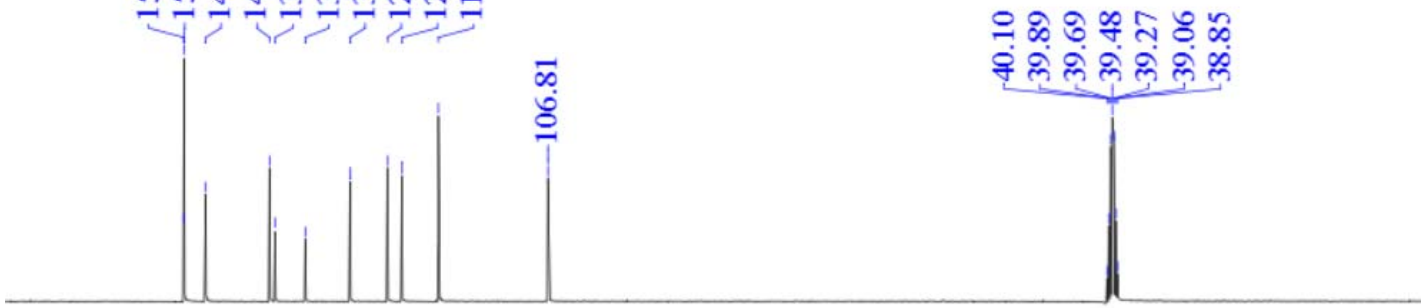

\begin{tabular}{|c|c|c|c|c|c|c|c|c|c|c|c|c|c|c|c|c|}
\hline & T & & 1 & & & T & & & & & & & & & & \\
\hline 170 & 160 & 150 & 140 & 130 & 120 & 110 & & & 80 & 70 & 60 & 50 & 40 & 30 & 20 & 10 \\
\hline
\end{tabular}

Figure S24. The ${ }^{1} \mathrm{H}$ and ${ }^{13} \mathrm{C}$ NMR spectra of 4,3'-pypzpy ligand. 

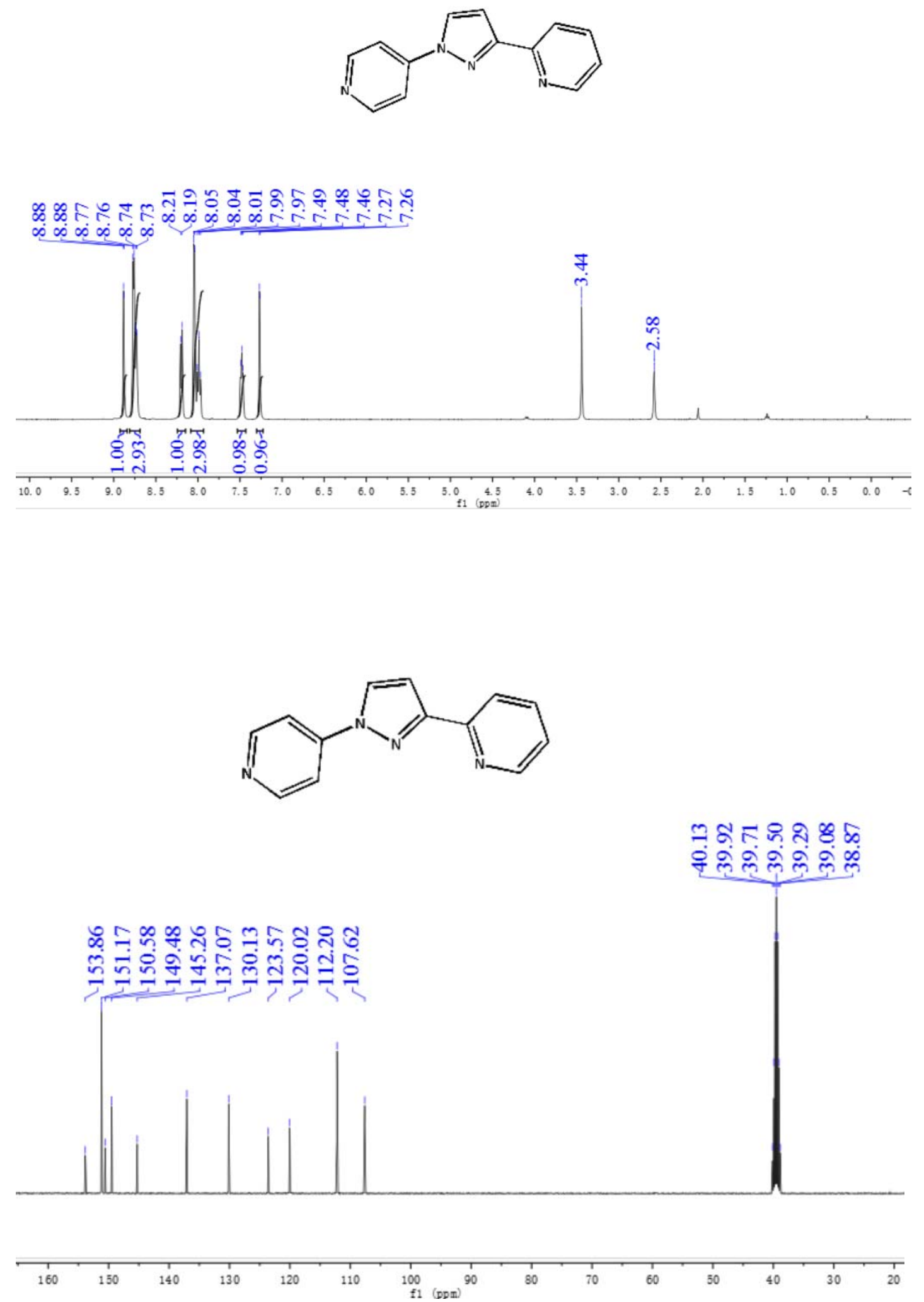

Figure S25. The ${ }^{1} \mathrm{H}$ and ${ }^{13} \mathrm{C}$ NMR spectra of 2,4'-pypzpy ligand. 


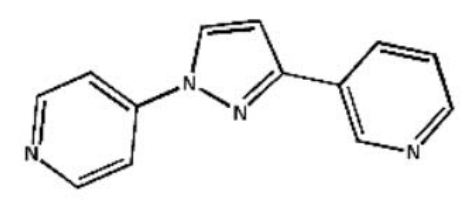

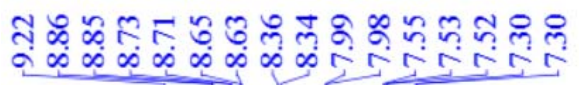
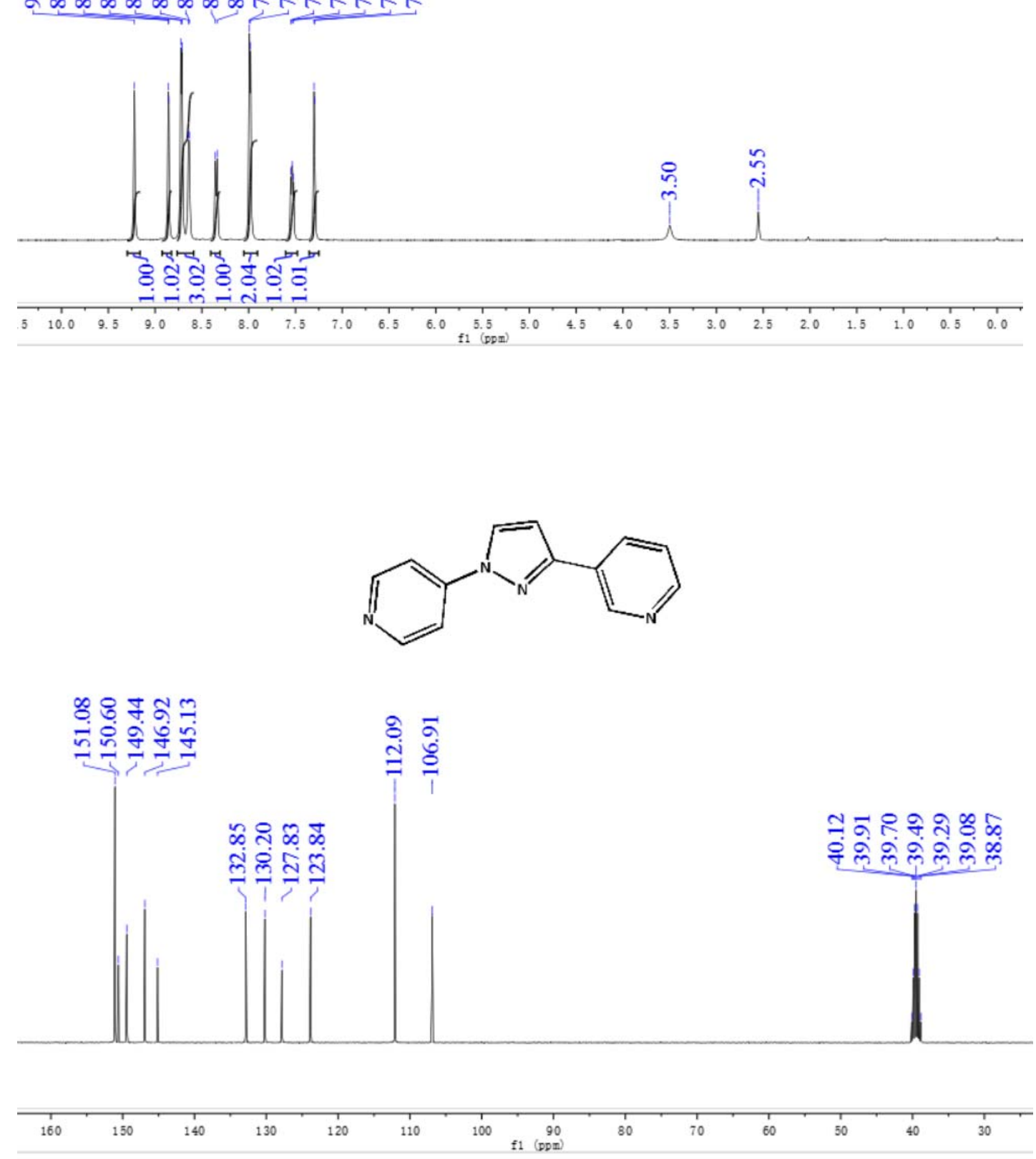

Figure S26. The ${ }^{1} \mathrm{H}$ and ${ }^{13} \mathrm{C}$ NMR spectra of 3,4'-pypzpy ligand. 

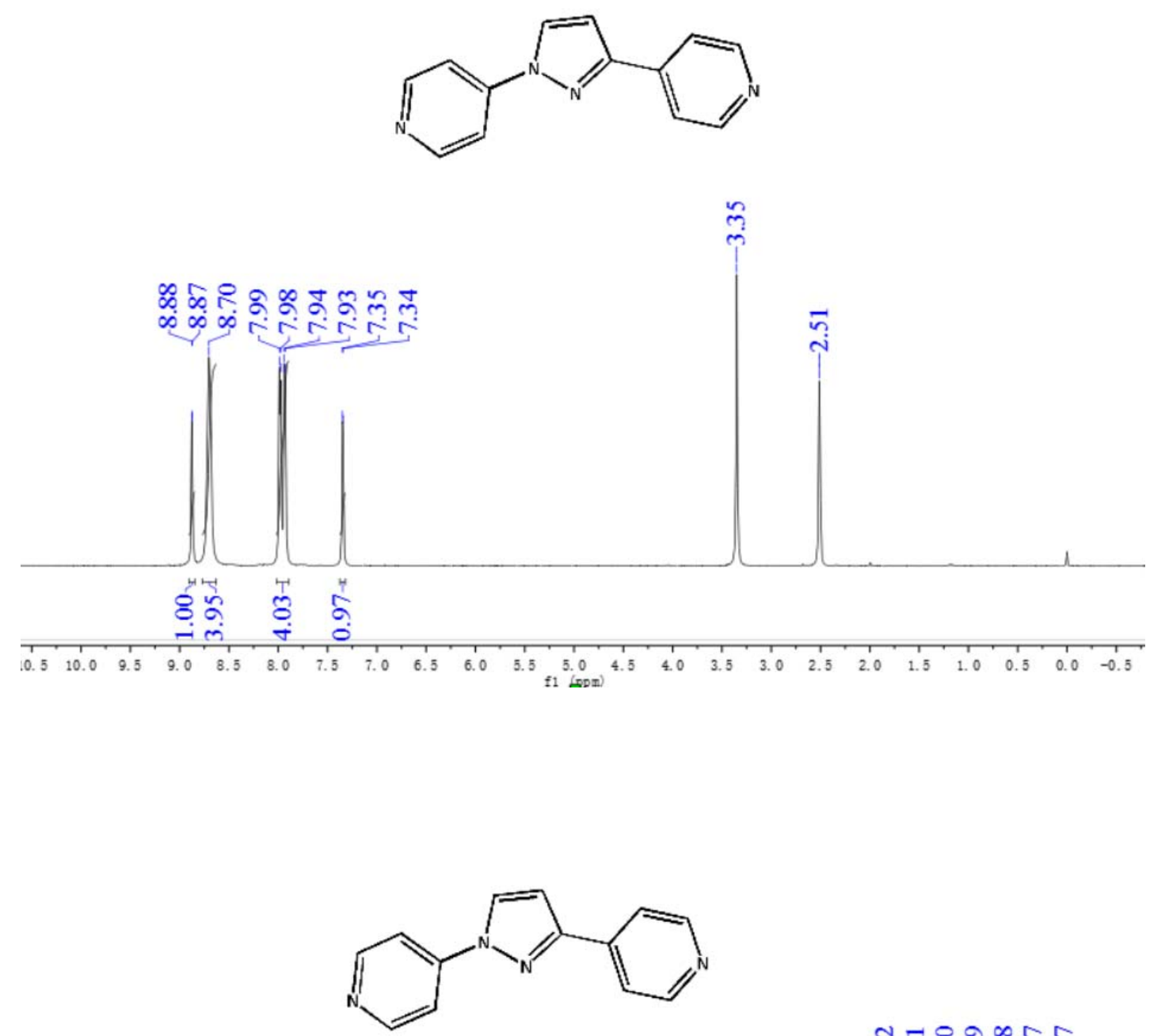

그ำำ ำ

के ले ले ले ले

กู่

กิํำ

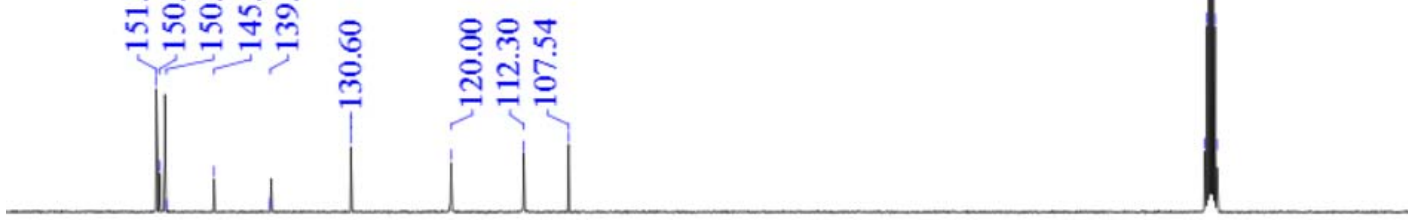

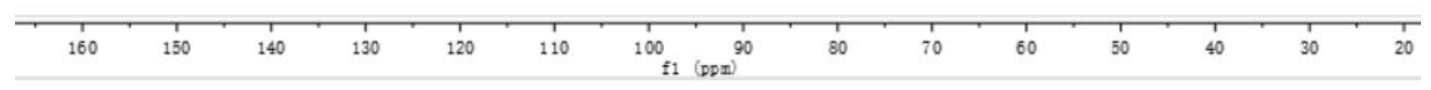

Figure S27. The ${ }^{1} \mathrm{H}$ and ${ }^{13} \mathrm{C}$ NMR spectra of 4,4'-pypzpy ligand. 